\title{
Existence and Global Attractivity of Positive Periodic Solutions for a Two-Species Competitive System with Stage Structure and Impulse
}

\author{
Changjin $X_{u^{1}}$ and Daxue Chen ${ }^{2}$ \\ ${ }^{1}$ Guizhou Key Laboratory of Economics System Simulation, School of Mathematics \\ and Statistics, Guizhou College of Finance and Economics, Guiyang 550004, China \\ ${ }^{2}$ Faculty of Science, Hunan Institute of Engineering, Xiangtan 411004, China
}

Correspondence should be addressed to Changjin Xu, xcj403@126.com

Received 10 August 2011; Accepted 17 September 2011

Academic Editor: Yuji Liu

Copyright (C) 2011 C. Xu and D. Chen. This is an open access article distributed under the Creative Commons Attribution License, which permits unrestricted use, distribution, and reproduction in any medium, provided the original work is properly cited.

\begin{abstract}
A class of nonautonomous two-species competitive system with stage structure and impulse is considered. By using the continuation theorem of coincidence degree theory, we derive a set of easily verifiable sufficient conditions that guarantee the existence of at least a positive periodic solution, and, by constructing a suitable Lyapunov functional, the uniqueness and global attractivity of the positive periodic solution are presented. Finally, an illustrative example is given to demonstrate the correctness of the obtained results.
\end{abstract}

\section{Introduction}

In recent years, with the increasing applications of theory of differential equations in mathematical ecology, various mathematical models have been proposed in the study of population [1-25]. But most of the previous results focused on the dynamical behaviors (including the stability, attractiveness, persistence, and periodicity of solution) of the systems which have fixed parameters and there is no impulse. Considering that harvest of many populations are not continuous and the periodic environmental factor, it is reasonable to investigate the systems with periodic coefficients and impulse. Impulsive differential systems display a combination of characteristics of both the continuous-time and discrete-time systems [26-30]. In 2006, Chen [1] studied the following non-autonomous almost periodic competitive two-species model with stage structure in one species: 


$$
\begin{gathered}
\dot{x}_{1}(t)=-a_{1}(t) x_{1}(t)+b_{1}(t) x_{2}(t), \\
\dot{x}_{2}(t)=a_{2}(t) x_{1}(t)-b_{2}(t) x_{2}(t)-c(t) x_{2}^{2}(t)-\beta_{1}(t) x_{2}(t) x_{2}(t) x_{3}(t), \\
\dot{x}_{3}(t)=x_{3}(t)\left[d(t)-e(t) x_{3}(t)-\beta_{2}(t) x_{2}(t)\right],
\end{gathered}
$$

where $x_{1}(t)$ and $x_{2}(t)$ are immature and mature population densities of one species, respectively; $x_{3}(t)$ represents the population density of another species; $a_{i}(t), b_{i}(t), \beta_{i}(t)(i=$ $1,2), c(t), d(t), e(t)$ are all continuous, almost periodic functions. The competition is between $x_{2}(t)$ and $x_{3}(t)$. Chen [1] obtained sufficient conditions for the existence of a unique, globally attractive, strictly positive almost periodic solution for system (1.1).

Considering that the harvest is an annual harvest pulse, to describe a system more accurately, we should consider the impulsive differential equation. Motivated by this point of view, we revised system (1.1) into the following form:

$$
\begin{gathered}
\dot{x}_{1}(t)=-a_{1}(t) x_{1}(t)+b_{1}(t) x_{2}(t), \quad t \neq t_{k}, \\
\dot{x}_{2}(t)=a_{2}(t) x_{2}(t)-b_{2}(t) x_{2}(t)-c(t) x_{2}^{2}(t)-\beta_{1}(t) x_{2}(t) x_{3}(t), \quad t \neq t_{k}, \\
\dot{x}_{3}(t)=x_{3}(t)\left[d(t)-e(t) x_{3}(t)-\beta_{2}(t) x_{2}(t)\right], \quad t \neq t_{k}, \\
\Delta x_{i}\left(t_{k}\right)=x_{i}\left(t_{k}^{+}\right)-x_{i}\left(t_{k}^{-}\right)=-\gamma_{i k} x_{i}(t k), \quad i=1,2,3, \quad k=1,2, \ldots, q,
\end{gathered}
$$

where $\Delta x_{i}\left(t_{k}\right)=x_{i}\left(t_{k}^{+}\right)-x_{i}\left(t_{k}^{-}\right)$are the impulses at moments $t_{k}$ and $t_{1}<t_{2}<\cdots$ is a strictly increasing sequence such that $\lim _{k \rightarrow \infty} t_{k}=+\infty ; x_{1}(t)$ and $x_{2}(t)$ are immature and mature population densities of one species, respectively, and $x_{3}(t)$ represents the population density of another species. The competition is between $x_{2}(t)$ and $x_{3}(t)$.

Throughout the paper, we always assume the following.

$\left(\mathrm{H}_{1}\right) a_{i}(t), b_{i}(t), \beta_{i}(t)(i=1,2), c(t), d(t), e(t)$ are all continuous $\omega$ periodic; that is, $a_{i}(t+$ $\omega)=a_{i}(t), b_{i}(t+\omega)=b_{i}(t), \beta_{i}(t+\omega)=\beta_{i}(t)(i=1,2), c(t+\omega)=c(t), d(t+\omega)=$ $d(t), e(t+\omega)=e(t)$ for any $t \in R$.

$\left(\mathrm{H}_{2}\right) a_{i}(t), b_{i}(t), \beta_{i}(t)(i=1,2), c(t), d(t), e(t)$ are all positive.

$\left(\mathrm{H}_{3}\right) 0<\gamma_{i k}<1, i=1,2,3$ for all $k \in N$, and there exists a positive integer $q$ such that $t_{k+q}=t_{k}+\omega, \gamma_{i(k+q)}=\gamma_{i k}, i=1,2,3$.

The principle object of this paper is by using Mawhin's continuation theorem of coincidence degree theory and by constructing the Lyapunov functions to investigate the stability and existence of periodic solutions of (1.2). To the best of my knowledge, it is the first time to deal with the existence and stability of periodic solutions of (1.2).

The organization of the paper is as follows. In Section 2, we introduce some notations and definitions and state some preliminary results needed in later sections. We then establish, in Section 3, some simple criteria for the existence of positive periodic solutions of system (1.2) by using the continuation theorem of coincidence degree theory proposed by Gaines and Mawhin [31]. The uniqueness and global attractivity of the positive periodic solution are presented in Section 4. In Section 5, an illustrative example is given to demonstrate the correctness of the obtained results. 


\section{Preliminaries}

We will introduce some notations and definitions and state some preliminary results. Consider the impulsive system

$$
\begin{gathered}
\dot{x}(t)=f(t, x), \quad t \neq t_{k}, \quad k=1,2, \ldots, \\
\left.\Delta x(t)\right|_{t=t_{k}}=I_{k}\left(x\left(t_{k}^{-}\right)\right),
\end{gathered}
$$

where $x \in R^{n}, f: R \times R^{n} \rightarrow R^{n}$ is continuous and $f(t+\omega, x)=f(t, x) ; I_{k}: R^{n} \rightarrow R^{n}$ are continuous, and there exists a positive integer $q$ such that $t_{k+q}=t_{k}+\omega, I_{k+q}(x)=I_{k}(x)$ with $t_{k} \in R, t_{k+1}>t_{k}, \lim _{k \rightarrow \infty}=\infty,\left.\Delta x(t)\right|_{t=t_{k}}=x\left(t_{k}^{+}\right)-x\left(t_{k}^{-}\right)$. For $t_{k} \neq 0(k=1,2, \ldots),[0, \omega] \cap\left\{t_{k}\right\}=$ $\left\{t_{1}, t_{2}, \ldots, t_{q}\right\}$. As we know, $\left\{t_{k}\right\}$ are called points of jump.

Let us recall some definitions. For the Canchy problem,

$$
\begin{gathered}
\dot{x}(t)=f(t, x), \quad t \in[0, \omega], \quad t \neq t_{k}, \\
\left.\Delta x(t)\right|_{t=t_{k}}=I_{k}\left(x\left(t_{k}^{-}\right)\right), \quad x(0)=x_{0} .
\end{gathered}
$$

Definition 2.1. A map $x:[0, \omega] \rightarrow R^{n}$ is said to be a solution of $(2.2)$, if it satisfied the following conditions:

(i) $x(t)$ is a piecewise continuous map with first-class discontinuity points in $t_{k} \cap[0, \omega]$, and at each discontinuity point it is continuous on the left;

(ii) $x(t)$ satisfies (2.2).

Definition 2.2. A map $x:[0, \omega] \rightarrow R^{n}$ is said to be an $\omega$ periodic solution of (2.1), if

(i) $x(t)$ satisfies (i) and (ii) of Definition 2.1 in the interval $[0, \omega]$ and

(ii) $x(t)$ satisfies $x(t+\omega-0)=x(t-0), t \in R$.

Obviously, if $x(t)$ is a solution of (2.2) defined on $[0, \omega]$, such that $x(0)=x(\omega)$, then, by the periodicity of (2.2) in $t$, the function $x^{*}(t)$ defined by

$$
x^{*}(t)=\left\{\begin{array}{l}
x(t-j \omega), \quad t \in[j \omega,(j+1) \omega] \backslash\left\{t_{k}\right\}, \\
x^{*}(t) \text { is left continuous at } t=t_{k}
\end{array}\right.
$$

is a $\omega$ periodic solution of $(2.1)$.

For system (1.2), seeking the periodic solutions is equivalent to seeking solutions of the following boundary value problem:

$$
\begin{gathered}
\dot{x}_{1}(t)=-a_{1}(t) x_{1}(t)+b_{1}(t) x_{2}(t), \quad t \neq t_{k}, t \in[0, \omega], k=1,2, \ldots, q, \\
\dot{x}_{2}(t)=a_{2}(t) x_{2}(t)-b_{2}(t) x_{2}(t)-c(t) x_{2}^{2}(t)-\beta_{1}(t) x_{2}(t) x_{3}(t), \quad t \neq t_{k}, t \in[0, \omega], k=1,2, \ldots, q, \\
\dot{x}_{3}(t)=x_{3}(t)\left[d(t)-e(t) x_{3}(t)-\beta_{2}(t) x_{2}(t)\right], \quad t \neq t_{k}, t \in[0, \omega], k=1,2, \ldots, q, \\
\Delta x_{i}\left(t_{k}\right)=x_{i}\left(t_{k}^{+}\right)-x_{i}\left(t_{k}^{-}\right)=-\gamma_{i k} x_{i}\left(t_{k}\right), \quad i=1,2,3, x_{i}(0)=x_{i}(\omega), k=1,2, \ldots, q .
\end{gathered}
$$




\section{Existence of Positive Periodic Solutions}

In this section, based on the Mawhin's continuation theorem, we shall study the existence of at least one periodic solution of (1.1). To do so, we shall make some preparations.

Let $X, Y$ be normed vector spaces; $L:$ Dom $L \subset X \rightarrow Y$ is a linear mapping; $N: X \rightarrow$ $Y$ is a continuous mapping. The mapping $L$ will be called a Fredholm mapping of index zero if $\operatorname{dimKer} L=$ codim Im $L<+\infty$ and $\operatorname{Im} L$ is closed in $Y$. If $L$ is a Fredholm mapping of index zero and there exist continuous projectors $P: X \rightarrow X$ and $Q: Y \rightarrow Y$ such that $\operatorname{Im} P=\operatorname{Ker} L, \operatorname{Im} L=\operatorname{Ker} Q=\operatorname{Im}(I-Q)$, it follows that $L \mid \operatorname{Dom} L \cap \operatorname{Ker} P:(I-P) X \rightarrow \operatorname{Im} L$ is invertible. We denote the inverse of that map by $K_{P}$. If $\Omega$ is an open bounded subset of $X$, the mapping $N$ will be called $L$-compact on $\bar{\Omega}$ if $Q N(\bar{\Omega})$ is bounded and $K_{P}(I-Q) N: \bar{\Omega} \rightarrow X$ is compact. Since $\operatorname{Im} Q$ is isomorphic to $\operatorname{Ker} L$, there exist isomorphisms $J: \operatorname{Im} Q \rightarrow \operatorname{Ker} L$.

Now we introduce Mawhin's continuation theorem [31] as follows.

Lemma 3.1 (Continuation Theorem [31]). Let L be a Fredholm mapping of index zero, and let $N$ be L-compact on $\bar{\Omega}$. Suppose

(a) for each $\lambda \in(0,1)$, every solution $x$ of $L x=\lambda N x$ is such that $x \notin \partial \Omega$.

(b) $Q N x \neq 0$ for each $x \in \operatorname{Ker} L \cap \partial \Omega$, and $\operatorname{deg}\{J Q N, \Omega \bigcap \partial \operatorname{Ker} L, 0\} \neq 0$.

Then the equation $L x=N x$ has at least one solution lying in $\operatorname{Dom} L \cap \bar{\Omega}$.

For convenience and simplicity in the following discussion, we always use the notations below throughout the paper:

$$
\bar{f}=\frac{1}{\omega} \int_{0}^{\omega} f(t) d t, \quad f^{L}=\min _{t \in[0, \omega]} f(t), \quad f^{M}=\max _{t \in[0, \omega]} f(t), \quad \overline{|f|}=\frac{1}{\omega} \int_{0}^{\omega}|f(t)| d t,
$$

where $f(t)$ is a $\omega$ continuous periodic function. For any nonnegative integer $p$, let $C^{(p)}\left[0, \omega ; t_{1}, t_{2}, \ldots, t_{q}\right]=\left\{x:[0, \omega] \rightarrow R^{m} \mid x^{(p)}(t)\right.$ exist for $t \neq t_{1}, \ldots, t_{q} ; x^{(p)}(t+0)$, and let $x^{(p)}(t-0)$ exist at $t_{1}, t_{2}, \ldots, t_{q}$, and $\left.x^{(j)}\left(t_{k}\right)=x^{(j)}\left(t_{k}-0\right), k=1, \ldots, m, j=0,1,2, \ldots, p\right\}$ with the norm $\|x\|_{p}=\max \left\{\sup _{t \in[0, \omega]}\left\|x^{(j)}(t)\right\|\right\}_{j=1^{\prime}}^{p}$, where $\|\cdot\|$ is any norm of $R^{m}$. It is easy to see that $C^{(p)}\left[0, \omega ; t_{1}, t_{2}, \ldots, t_{q}\right]$ is a Banach space.

Now we are now in a position to state and prove the existence of periodic solutions of (2.4).

Theorem 3.2. In addition to $\left(\mathrm{H}_{1}\right),\left(\mathrm{H}_{2}\right),\left(\mathrm{H}_{3}\right)$, assume further that the following hold:

$$
\begin{aligned}
& \left(\mathrm{H}_{4}\right) \min \left\{P_{1}, P_{2}, P_{3}\right\}>0, \\
& \left(\mathrm{H}_{5}\right) \bar{a}_{1} \omega>\sum_{k=1}^{q} \ln \left(1-\gamma_{1 k}\right), \\
& \left(\mathrm{H}_{6}\right) \bar{d} \omega+\sum_{k=1}^{q} \ln \left(1-\gamma_{3 k}\right)>\bar{\beta}_{2} \omega e^{B_{4}}, \quad \bar{\beta}_{1} \bar{\beta}_{2} \neq \overline{c e},
\end{aligned}
$$


where

$$
\begin{aligned}
& P_{1}=\overline{\left|a_{2}-b_{2}\right|} \omega+\sum_{k=1}^{q} \ln \left(1-\gamma_{2 k}\right)-\bar{c} \omega e^{B_{6}}, \\
& P_{2}=\overline{\left|a_{2}-b_{2}\right|} \omega+\sum_{k=1}^{q} \ln \left(1-\gamma_{2 k}\right)-\bar{\beta}_{1} \omega e^{B_{29}}, \\
& P_{3}=\overline{\left|a_{2}-b_{2}\right|} \omega+\sum_{k=1}^{q} \ln \left(1-\gamma_{2 k}\right)-\bar{c} \omega,
\end{aligned}
$$

and $B_{4}, B_{6}, B_{29}$ are defined by (3.27), (3.32), and (3.61), respectively. Then the system (1.2) has at least a $\omega$ periodic solution.

Proof. According to the discussion above in Section 2, we need only to prove that the boundary value problem (2.4) has a solution. Since solutions of (2.4) remained positive for all $t \geq 0$, we let

$$
u_{1}(t)=\ln \left[x_{1}(t)\right], \quad u_{2}(t)=\ln \left[x_{2}(t)\right], \quad u_{3}(t)=\ln \left[x_{3}(t)\right],
$$

then system (2.4) can be translated to

$$
\begin{gathered}
\dot{u}_{1}(t)=-a_{1}(t)+b_{1}(t) e^{\left(u_{2}(t)-u_{1}(t)\right)}, \quad t \neq t_{k}, t \in[0, \omega], k=1,2, \ldots, q, \\
\dot{u}_{2}(t)=a_{2}(t)-b_{2}(t)-c(t) e^{u_{2}(t)}-\beta_{1}(t) e^{u_{3}(t)}, \quad t \neq t_{k}, t \in[0, \omega], k=1,2, \ldots, q, \\
\dot{u}_{3}(t)=d(t)-e(t) e^{u_{3}(t)}-\beta_{2}(t) e^{u_{2}(t)}, \quad t \neq t_{k}, t \in[0, \omega], k=1,2, \ldots, q, \\
\Delta u_{i}\left(t_{k}\right)=\ln \left(1-\gamma_{i k}\right), \quad i=1,2,3, \quad u_{i}(0)=u_{i}(\omega) .
\end{gathered}
$$

It is easy to see that if system (3.5) has one $\omega$ periodic solution $\left(u_{1}^{*}(t), u_{2}^{*}(t), u_{3}^{*}(t)\right)^{T}$, then $\left(x_{1}^{*}(t), x_{2}^{*}(t), x_{3}^{*}(t)\right)^{T}=\left(e^{u_{1}^{*}(t)}, e^{u_{2}^{*}(t)}, e^{u_{3}^{*}(t)}\right)^{T}$ is a positive solution of system (1.2). Therefore, to complete the proof, it suffices to show that system (3.5) has at least one $\omega$ periodic solution.

In order to use the continuation theorem of coincidence degree theory, we take

$$
X=\left\{u \in C\left[0, \omega ; t_{1}, t_{2}, \ldots, t_{q}\right]\right\}, \quad Y=X \times R^{3 \times(q+1)} .
$$

Then $X$ is a Banach space with norm $\|\cdot\|_{0}$, and $Y$ is also a Banch space with norm $\|z\|=$ $\|x\|_{0}+\|y\|, x \in X, Y \in R^{3 q}$. 
Let the following hold:

$$
\begin{aligned}
& \operatorname{dom} L=\left\{x=\left(u_{1}, u_{2}, u_{3}\right)^{T} \in C[0, \omega] ; t_{1}, t_{2}, \ldots, t_{q}\right\} \text {, } \\
& L: \operatorname{Dom} L \subset X \longrightarrow Y, \quad x \longrightarrow\left(x^{\prime}, \Delta x\left(t_{k}\right)_{k=1}^{q}\right), \\
& N: X \longrightarrow Y \text {, } \\
& N u=\left(\left(\begin{array}{c}
-a_{1}(t)+b_{1}(t) \exp \left(u_{2}(t)-u_{1}(t)\right) \\
a_{2}(t)-b_{2}(t)-c(t) e^{u_{2}(t)}-\beta_{1}(t) e^{u_{3}(t)} \\
d(t)-e(t) e^{u_{3}(t)}-\beta_{2}(t) e^{u_{2}(t)}
\end{array}\right),\left(\begin{array}{c}
\ln \left(1-\gamma_{11}\right) \\
\ln \left(1-\gamma_{21}\right) \\
\ln \left(1-\gamma_{31}\right)
\end{array}\right),\right. \\
& \left.\left(\begin{array}{l}
\ln \left(1-\gamma_{12}\right) \\
\ln \left(1-\gamma_{22}\right) \\
\ln \left(1-\gamma_{32}\right)
\end{array}\right), \ldots,\left(\begin{array}{c}
\ln \left(1-\gamma_{1 q}\right) \\
\ln \left(1-\gamma_{2 q}\right) \\
\ln \left(1-\gamma_{3 q}\right)
\end{array}\right), 0\right)
\end{aligned}
$$

Obviously,

$$
\begin{aligned}
\operatorname{Ker} L & =\left\{u: u(t)=h \in R^{3}, t \in[0, \omega]\right\}, \\
\operatorname{Im} L & =\left\{z=\left(f, a_{1}, a_{2}, \ldots, a_{q}, d\right) \in Y: \int_{0}^{\omega} f(s) d s+\sum_{k=1}^{q} a_{k}+d=0\right\} \\
& =X \times R^{3 \times q} \times\{0\},
\end{aligned}
$$

$\operatorname{dimKer} L=3=\operatorname{codim} I m L$.

So, $\operatorname{Im} L$ is closed in $Y$; $L$ is a Fredholm mapping of index zero. Define two projectors

$$
\begin{gathered}
P x=\frac{1}{\omega} \int_{0}^{\omega} x(t) d t \\
Q z=Q\left(f, a_{1}, a_{2}, \ldots, a_{q}, d\right)=\left(\frac{1}{\omega}\left[\int_{0}^{\omega} f(s) d s+\sum_{k=1}^{q} a_{k}+d,\right], 0,0, \ldots, 0\right) .
\end{gathered}
$$

It is easy to show that $P$ and $Q$ are continuous and satisfy $\operatorname{Im} P=\operatorname{Ker} L, \operatorname{Im} L=\operatorname{Ker} Q=$ $\operatorname{Im}(I-Q)$.

Further, by direct computation, we can find that the inverse $K_{P}$ of $L, K_{P}: \operatorname{Im} L \rightarrow$ Ker $P \cap$ Dom $L$ has the following form:

$$
K_{P}(z)=\int_{0}^{t} f(s) d s+\sum_{t_{k}<t} a_{k}-\frac{1}{\omega} \int_{0}^{\omega} \int_{0}^{t} f(s) d s d t-\sum_{k=1}^{q} a_{k}+\frac{1}{\omega} \sum_{k=1}^{q} a_{k} t_{k} .
$$


Moreover, it is easy to check that

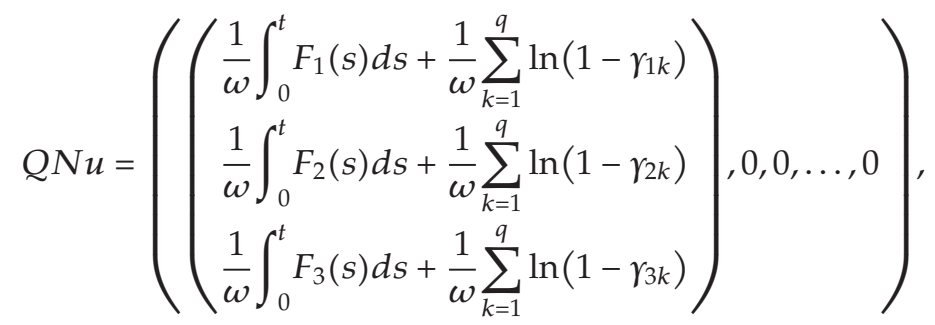

$$
\begin{aligned}
& K_{P}(I-Q) N u=\left(\begin{array}{l}
\int_{0}^{t} F_{1}(s) d s+\sum_{t>t_{k}} \ln \left(1-\gamma_{1 k}\right) \\
\int_{0}^{t} F_{2}(s) d s+\sum_{t>t_{k}} \ln \left(1-\gamma_{2 k}\right) \\
\int_{0}^{t} F_{3}(s) d s+\sum_{t>t_{k}} \ln \left(1-\gamma_{3 k}\right)
\end{array}\right) \\
& -\left(\begin{array}{c}
\frac{1}{\omega} \int_{0}^{\omega} \int_{0}^{t} F_{1}(s) d s d t-\sum_{k=1}^{q} \ln \left(1-\gamma_{1 k}\right)+\frac{1}{\omega} \sum_{k=1}^{q} \ln \left(1-\gamma_{1 k}\right) \\
\frac{1}{\omega} \int_{0}^{\omega} \int_{0}^{t} F_{2}(s) d s d t-\sum_{k=1}^{q} \ln \left(1-\gamma_{2 k}\right)+\frac{1}{\omega} \sum_{k=1}^{q} \ln \left(1-\gamma_{2 k}\right) \\
\frac{1}{\omega} \int_{0}^{\omega} \int_{0}^{t} F_{3}(s) d s d t-\sum_{k=1}^{q} \ln \left(1-\gamma_{3 k}\right)+\frac{1}{\omega} \sum_{k=1}^{q} \ln \left(1-\gamma_{3 k}\right)
\end{array}\right) \\
& -\left(\frac{t}{\omega}-\frac{1}{2}\right)\left(\begin{array}{l}
\int_{0}^{\omega} F_{1}(s) d s+\sum_{k=1}^{q} \ln \left(1-\gamma_{1 k}\right) \\
\int_{0}^{\omega} F_{2}(s) d s+\sum_{k=1}^{q} \ln \left(1-\gamma_{2 k}\right) \\
\int_{0}^{\omega} F_{3}(s) d s+\sum_{k=1}^{q} \ln \left(1-\gamma_{3 k}\right)
\end{array}\right),
\end{aligned}
$$

where

$$
\begin{gathered}
F_{1}(s)=-a_{1}(s)+b_{1}(s) e^{\left(u_{2}(s)-u_{1}(s)\right)}, \\
F_{2}(s)=a_{2}(s)-b_{2}(s)-c(s) e^{u_{2}(s)}-\beta_{1}(s) e^{u_{3}(s)}, \\
F_{2}(s)=d(s)-e(s) e^{u_{3}(s)}-\beta_{2}(s) e^{u_{2}(s)} .
\end{gathered}
$$

Obviously, $Q N$ and $K_{P}(I-Q) N$ are continuous. Using the Ascoli-Arzela theorem, it is not difficult to show that $\overline{K_{P}(I-Q) N(\bar{\Omega})}$ is compact for any open bounded set $\Omega \subset X$. Moreover, $Q N(\bar{\Omega})$ is bounded. Thus, $N$ is $L$-compact on $\bar{\Omega}$ with any open bounded set $\Omega \subset X$. 
Now we are at the point to search for an appropriate open, bounded subset $\Omega$ for the application of the continuation theorem. Corresponding to the operator equation $L u=$ $\lambda N u, \lambda \in(0,1)$, we have

$$
\begin{gathered}
\dot{u}_{1}(t)=\lambda\left[-a_{1}(t)+b_{1}(t) e^{\left(u_{2}(t)-u_{1}(t)\right)}\right], \quad t \neq t_{k}, t \in[0, \omega], k=1,2, \ldots, q, \\
\dot{u}_{2}(t)=\lambda\left[a_{2}(t)-b_{2}(t)-c(t) e^{u_{2}(t)}-\beta_{1}(t) e^{u_{3}(t)}\right], \quad t \neq t_{k}, t \in[0, \omega], k=1,2, \ldots, q, \\
\left.\dot{u}_{3}(t)=\lambda\left[d_{1}(t)-e(t) e^{u_{3}(t)}-\beta_{2}(t) e^{u_{2}(t)}\right)\right], \quad t \neq t_{k}, t \in[0, \omega], k=1,2, \ldots, q, \\
\Delta u_{i}\left(t_{k}\right)=\lambda \ln \left(1-\gamma_{i k}\right), \quad i=1,2,3, \quad u_{i}(0)=u_{i}(\omega) .
\end{gathered}
$$

Suppose that $u(t)=\left(u_{1}(t), u_{2}(t), u_{3}(t)\right)^{T} \in X$ is an arbitrary solution of system (3.13) for a certain $\lambda \in(0,1)$, integrating both sides of (3.13) over the interval $[0, \omega]$ with respect to $t$, we obtain

$$
\begin{gathered}
\int_{0}^{\omega}\left[b_{1}(t) e^{\left(u_{2}(t)-u_{1}(t)\right)}\right] d t+\sum_{k=1}^{q} \ln \left(1-\gamma_{1 k}\right)=\int_{0}^{\omega} a_{1}(t) d t \\
\int_{0}^{\omega}\left[c(t) e^{u_{2}(t)}+\beta_{1}(t) e^{u_{3}(t)}\right] d t=\int_{0}^{\omega}\left(a_{2}(t)-b_{2}(t)\right) d t+\sum_{k=1}^{q} \ln \left(1-\gamma_{2 k}\right), \\
\int_{0}^{\omega}\left[e(t) e^{u_{3}(t)}+\beta_{2}(t) e^{u_{2}(t)}\right] d t=\int_{0}^{\omega} d(t) d t+\sum_{k=1}^{q} \ln \left(1-\gamma_{3 k}\right) .
\end{gathered}
$$

From (3.13) and (3.14), we obtain

$$
\begin{aligned}
\int_{0}^{\omega}\left|\dot{u}_{1}(t)\right| d t & <\int_{0}^{\omega} a_{1}(t) d t+\int_{0}^{\omega}\left[b_{1}(t) e^{\left(u_{2}(t)-u_{1}(t)\right)}\right] d t \\
& =2 \bar{a}_{1} \omega-\sum_{k=1}^{q} \ln \left(1-\gamma_{1 k}\right), \\
\int_{0}^{\omega}\left|\dot{u}_{2}(t)\right| d t & <\int_{0}^{\omega}\left[a_{2}(t)+b_{2}(t)\right] d t+\int_{0}^{\omega}\left[c(t) e^{u_{2}(t)}+\beta_{1}(t) e^{\left.u_{3}(t)\right)}\right] d t \\
& =2 \bar{a}_{2} \omega+\sum_{k=1}^{q} \ln \left(1-\gamma_{2 k}\right), \\
\int_{0}^{\omega}\left|\dot{u}_{3}(t)\right| d t & \left.<\int_{0}^{\omega} d(t) d t+\int_{0}^{\omega}\left[e(t) e^{u_{3}(t)}+\beta_{2}(t) e^{u_{2}(t)}\right)\right] d t \\
& =2 \bar{d} \omega+\sum_{k=1}^{q} \ln \left(1-\gamma_{3 k}\right) .
\end{aligned}
$$


Let the following hold:

$$
u_{i}\left(\xi_{i}\right)=\min _{t \in[0, \omega]} u_{i}(t), \quad u_{i}\left(\eta_{i}\right)=\max _{t \in[0, \omega]} u_{i}(t), \quad i=1,2,3
$$

From the second and the third equations of (3.14), we can obtain

$$
\begin{gathered}
\overline{\left|a_{2}-b_{2}\right|} \omega+\sum_{k=1}^{q} \ln \left(1-\gamma_{2 k}\right)>\int_{0}^{\omega} c(t) e^{u_{2}(t)} d t \geq \int_{0}^{\omega} c(t) e^{u_{2}\left(\xi_{2}\right)} d t \\
\bar{d} \omega+\sum_{k=1}^{q} \ln \left(1-\gamma_{3 k}\right)>\int_{0}^{\omega} e(t) e^{u_{3}(t)} d t \geq \int_{0}^{\omega} e(t) e^{u_{3}\left(\xi_{3}\right)} d t=\bar{e} \omega e^{u_{3}\left(\xi_{3}\right)},
\end{gathered}
$$

then

$$
\begin{gathered}
u_{2}\left(\xi_{2}\right)<\ln \left[\frac{\overline{\left|a_{2}-b_{2}\right|} \omega+\sum_{k=1}^{q} \ln \left(1-\gamma_{2 k}\right)}{\bar{c} \omega}\right], \\
u_{3}\left(\xi_{3}\right)<\ln \left[\frac{\bar{d} \omega+\sum_{k=1}^{q} \ln \left(1-\gamma_{3 k}\right)}{\bar{e} \omega}\right] .
\end{gathered}
$$

Thus

$$
\begin{aligned}
u_{2}(t) & =u_{2}\left(\xi_{2}\right)+\int_{\xi_{2}}^{t} \dot{u}_{2}(t) d t \leq u_{2}\left(\xi_{2}\right)+\int_{0}^{\omega}\left|\dot{u}_{2}(t)\right| d t \\
& <\ln \left[\frac{\overline{\left|a_{2}-b_{2}\right|} \omega+\sum_{k=1}^{q} \ln \left(1-\gamma_{2 k}\right)}{\bar{c} \omega}\right]+2 \bar{a}_{2} \omega+\sum_{k=1}^{q} \ln \left(1-\gamma_{2 k}\right)=: B_{1} .
\end{aligned}
$$

In the following, we will consider four cases.

Case 1 (if $u_{1}(t)>0, u_{2}(t)>0$ ). From the first equation of (3.14), we have

$$
\begin{aligned}
& \bar{a}_{1} \omega<\int_{0}^{\omega} b_{1}(t) e^{u_{2}(t)} d t+\sum_{k=1}^{q} \ln \left(1-\gamma_{1 k}\right) \leq \bar{b}_{1} \omega e^{u_{2}\left(\eta_{2}\right)}+\sum_{k=1}^{q} \ln \left(1-\gamma_{3 k}\right), \\
& \bar{a}_{1} \omega>\int_{0}^{\omega} b_{1}(t) e^{-u_{1}(t)} d t+\sum_{k=1}^{q} \ln \left(1-\gamma_{1 k}\right) \geq \int_{0}^{\omega} b_{1}(t) e^{-u_{1}\left(\eta_{1}\right)} d t+\sum_{k=1}^{q} \ln \left(1-\gamma_{1 k}\right),
\end{aligned}
$$


that is,

$$
\begin{aligned}
& u_{2}\left(\eta_{2}\right)>\ln \left[\frac{\bar{a}_{1} \omega-\sum_{k=1}^{q} \ln \left(1-\gamma_{1 k}\right)}{\bar{b}_{1} \omega}\right], \\
& u_{1}\left(\eta_{1}\right)>\ln \left[\frac{\bar{b}_{1} \omega}{\bar{a}_{1} \omega-\sum_{k=1}^{q} \ln \left(1-\gamma_{1 k}\right)}\right] .
\end{aligned}
$$

Then

$$
\begin{aligned}
u_{2}(t) & =u_{2}\left(\eta_{2}\right)-\int_{t}^{\eta_{2}} \dot{u}_{2}(t) d t \geq u_{2}\left(\eta_{2}\right)-\int_{0}^{\omega}\left|\dot{u}_{2}(t)\right| d t \\
& \geq \ln \left[\frac{\bar{a}_{1} \omega-\sum_{k=1}^{q} \ln \left(1-\gamma_{1 k}\right)}{\bar{b}_{1} \omega}\right]-2 \bar{a}_{2} \omega-\sum_{k=1}^{q} \ln \left(1-\gamma_{2 k}\right)=: B_{2}, \\
u_{1}(t) & =u_{1}\left(\eta_{1}\right)-\int_{t}^{\eta_{1}} \dot{u}_{1}(t) d t \geq u_{1}\left(\eta_{1}\right)-\int_{0}^{\omega}\left|\dot{u}_{1}(t)\right| d t \\
& \geq \ln \left[\frac{\bar{b}_{1} \omega}{\bar{a}_{1} \omega-\sum_{k=1}^{q} \ln \left(1-\gamma_{1 k}\right)}\right]-2 \bar{a}_{1} \omega-\sum_{k=1}^{q} \ln \left(1-\gamma_{1 k}\right)=: B_{3} .
\end{aligned}
$$

Thus, from (3.22) and (3.25), we obtain

$$
\left|u_{2}(t)\right| \leq \max \left\{\left|B_{1}\right|,\left|B_{2}\right|\right\}=: B_{4} .
$$

By the first and the third equations of (3.14), we get

$$
\begin{gathered}
\int_{0}^{\omega}\left[b_{1}(t) e^{B_{4}-u_{1}\left(\xi_{1}\right)}\right] d t+\sum_{k=1}^{q} \ln \left(1-\gamma_{1 k}\right)>\bar{a}_{1} \omega, \\
\int_{0}^{\omega}\left[e(t) e^{u_{3}\left(\eta_{3}\right)}+\beta_{2}(t) e^{B_{4}}\right] d t>\bar{d} \omega+\sum_{k=1}^{q} \ln \left(1-\gamma_{3 k}\right),
\end{gathered}
$$

then

$$
\begin{aligned}
& u_{1}\left(\xi_{1}\right)<\ln \left[\frac{\bar{b}_{1} \omega e^{B_{4}}}{\bar{a}_{1} \omega-\sum_{k=1}^{q} \ln \left(1-\gamma_{1 k}\right)}\right] \\
& u_{3}\left(\eta_{3}\right)>\ln \left[\frac{\bar{d} \omega+\sum_{k=1}^{q} \ln \left(1-\gamma_{3 k}\right)-\beta_{2} \omega e^{B_{4}}}{\bar{e} \omega}\right] .
\end{aligned}
$$


International Journal of Differential Equations

From (3.15), (3.17), (3.21), and (3.30), we have

$$
\begin{aligned}
u_{1}(t) & =u_{1}\left(\xi_{1}\right)+\int_{\xi_{1}}^{t} \dot{u}_{1}(t) d t \leq u_{1}\left(\xi_{1}\right)+\int_{0}^{\omega}\left|\dot{u}_{1}(t)\right| d t \\
& <\ln \left[\frac{\bar{b}_{1} \omega e^{B_{4}}}{\bar{a}_{1} \omega-\sum_{k=1}^{q} \ln \left(1-\gamma_{1 k}\right)}\right]+2 \bar{a}_{1} \omega+\sum_{k=1}^{q} \ln \left(1-\gamma_{1 k}\right)=: B_{5}, \\
u_{3}(t) & =u_{3}\left(\xi_{3}\right)+\int_{\xi_{3}}^{t} \dot{u}_{3}(t) d t \leq u_{3}\left(\xi_{3}\right)+\int_{0}^{\omega}\left|\dot{u}_{3}(t)\right| d t \\
& <\ln \left[\frac{\bar{d} \omega+\sum_{k=1}^{q} \ln \left(1-\gamma_{3 k}\right)}{\bar{e} \omega}\right]+2 \bar{d} \omega+\sum_{k=1}^{q} \ln \left(1-\gamma_{3 k}\right)=: B_{6}, \\
u_{3}(t) & =u_{3}\left(\eta_{3}\right)-\int_{t}^{\eta_{3}} \dot{u}_{3}(t) d t \geq u_{3}\left(\eta_{3}\right)-\int_{0}^{\omega}\left|\dot{u}_{3}(t)\right| d t \\
& >\ln \left[\frac{\bar{d} \omega+\sum_{k=1}^{q} \ln \left(1-\gamma_{3 k}\right)-\bar{\beta}_{2} \omega e^{B_{4}}}{\bar{e} \omega}\right]-2 \bar{d} \omega+\sum_{k=1}^{q} \ln \left(1-\gamma_{3 k}\right)=: B_{7} .
\end{aligned}
$$

Thus,

$$
\left|u_{1}(t)\right| \leq B_{5}, \quad\left|u_{3}(t)\right| \max \left\{\left|B_{6}\right|,\left|B_{7}\right|\right\}=: B_{8} .
$$

Case 2 (if $\left.u_{1}(t)>0, u_{2}(t)<0\right)$. By the first equation of $(3.14)$, we have

$$
\bar{a}_{1} \omega<\int_{0}^{\omega} b_{1}(t) e^{u_{2}(t)} d t+\sum_{k=1}^{q} \ln \left(1-\gamma_{1 k}\right) \leq \bar{b}_{1} \omega e^{u_{2}\left(\eta_{2}\right)}+\sum_{k=1}^{q} \ln \left(1-\gamma_{3 k}\right),
$$

namely,

$$
u_{2}\left(\eta_{2}\right)>\ln \left[\frac{\bar{a}_{1} \omega-\sum_{k=1}^{q} \ln \left(1-\gamma_{1 k}\right)}{\bar{b}_{1} \omega}\right] .
$$

Then

$$
\begin{aligned}
u_{2}(t) & =u_{2}\left(\eta_{2}\right)-\int_{t}^{\eta_{2}} \dot{u}_{2}(t) d t \geq u_{2}\left(\eta_{2}\right)-\int_{0}^{\omega}\left|\dot{u}_{2}(t)\right| d t \\
& \geq \ln \left[\frac{\bar{a}_{1} \omega-\sum_{k=1}^{q} \ln \left(1-\gamma_{1 k}\right)}{\bar{b}_{1}}\right]-2 \bar{a}_{2} \omega-\sum_{k=1}^{q} \ln \left(1-\gamma_{2 k}\right)=: B_{2} .
\end{aligned}
$$


From (3.22) and (3.37), we obtain

$$
\left|u_{2}(t)\right| \leq \max \left\{\left|B_{1}\right|,\left|B_{2}\right|\right\}=: B_{3} .
$$

By the first equation of (3.14), we also have

$$
\begin{aligned}
& \int_{0}^{\omega} \frac{b_{1}(t) e^{B_{3}}}{e^{u_{1}(t)}} d t+\sum_{k=1}^{q} \ln \left(1-\gamma_{1 k}\right)>\bar{a}_{1} \omega, \\
& \int_{0}^{\omega} \frac{b_{1}(t) e^{-B_{3}}}{e^{u_{1}(t)}} d t+\sum_{k=1}^{q} \ln \left(1-\gamma_{1 k}\right)<\bar{a}_{1} \omega,
\end{aligned}
$$

Then

$$
\begin{aligned}
& \frac{\bar{b}_{1} \omega e^{B_{3}}}{e^{u_{1}\left(\xi_{1}\right)}}+\sum_{k=1}^{q} \ln \left(1-\gamma_{1 k}\right)>\bar{a}_{1} \omega, \\
& \frac{\bar{b}_{1} \omega e^{-B_{3}}}{e^{u_{1}\left(\eta_{1}\right)}}+\sum_{k=1}^{q} \ln \left(1-\gamma_{1 k}\right)<\bar{a}_{1} \omega .
\end{aligned}
$$

that is,

$$
\begin{aligned}
& u_{1}\left(\xi_{1}\right)<\ln \left[\frac{\bar{b}_{1} \omega e^{B_{3}}}{\bar{a}_{1} \omega-\sum_{k=1}^{q} \ln \left(1-\gamma_{1 k}\right)}\right]=: B_{8}, \\
& u_{1}\left(\eta_{1}\right)<\ln \left[\frac{\bar{b}_{1} \omega e^{B_{3}}}{\bar{a}_{1} \omega-\sum_{k=1}^{q} \ln \left(1-\gamma_{1 k}\right)}\right]=: B_{9} .
\end{aligned}
$$

Thus,

$$
\begin{aligned}
u_{1}(t) & =u_{1}\left(\xi_{1}\right)+\int_{\xi_{1}}^{t} \dot{u}_{1}(t) d t \leq u_{1}\left(\xi_{1}\right)+\int_{0}^{\omega}\left|\dot{u}_{1}(t)\right| d t \\
& <B_{8}+2 \bar{a}_{1} \omega-\sum_{k=1}^{q} \ln \left(1-\gamma_{1 k}\right)=: B_{10}, \\
u_{1}(t) & =u_{1}\left(\eta_{1}\right)+\int_{t}^{\eta_{1}} \dot{u}_{1}(t) d t \geq u_{1}\left(\eta_{1}\right)+\int_{0}^{\omega}\left|\dot{u}_{1}(t)\right| d t \\
& >B_{9}-2 \bar{a}_{1} \omega-\sum_{k=1}^{q} \ln \left(1-\gamma_{1 k}\right)=: B_{11} .
\end{aligned}
$$

From (3.42), we have

$$
\mid u_{1}(t) \leq \max \left\{\left|B_{10}\right|,\left|B_{11}\right|\right\}=: B_{12}
$$


International Journal of Differential Equations

By the second equation of (3.14), we have

$$
\begin{aligned}
& \int_{0}^{\omega} c(t) e^{B_{3}} d t+\int_{0}^{\omega} \beta_{1}(t) e^{u_{3}(t)} d t>\overline{\left|a_{2}-b_{2}\right|} \omega+\sum_{k=1}^{q} \ln \left(1-\gamma_{2 k}\right), \\
& \int_{0}^{\omega} c(t) e^{-B_{3}} d t+\int_{0}^{\omega} \beta_{1}(t) e^{u_{3}(t)} d t<\overline{\left|a_{2}-b_{2}\right|} \omega+\sum_{k=1}^{q} \ln \left(1-\gamma_{2 k}\right) .
\end{aligned}
$$

Then

$$
\begin{gathered}
\bar{c} \omega e^{B_{3}}+\bar{\beta}_{1} \omega e^{u_{3}\left(\eta_{3}\right)}>\overline{\left|a_{2}-b_{2}\right|} \omega+\sum_{k=1}^{q} \ln \left(1-\gamma_{2 k}\right), \\
\bar{c} \omega e^{-B_{3}}+\bar{\beta}_{1} \omega e^{u_{3}\left(\xi_{3}\right)}<\overline{\left|a_{2}-b_{2}\right|} \omega+\sum_{k=1}^{q} \ln \left(1-\gamma_{2 k}\right)=: B_{13},
\end{gathered}
$$

that is,

$$
\begin{gathered}
u_{3}\left(\eta_{3}\right)>\ln \left[\frac{\overline{\left|a_{2}-b_{2}\right|} \omega+\sum_{k=1}^{q} \ln \left(1-\gamma_{2 k}\right)-\bar{c} \omega e^{B_{6}}}{\bar{\beta}_{1} \omega}\right] \\
u_{3}\left(\xi_{3}\right)<\ln \left[\frac{\mid \overline{\left|a_{2}-b_{2}\right|} \omega+\sum_{k=1}^{q} \ln \left(1-\gamma_{2 k}\right)-\bar{c} \omega e^{-B_{3}}}{\bar{\beta}_{1} \omega}\right]=: B_{14} .
\end{gathered}
$$

Therefore, we get

$$
\begin{aligned}
u_{3}(t) & =u_{3}\left(\xi_{3}\right)+\int_{\xi_{3}}^{t} \dot{u}_{3}(t) d t \leq u_{3}\left(\xi_{3}\right)+\int_{0}^{\omega}\left|\dot{u}_{3}(t)\right| d t \\
& <B_{14}+2 \bar{d}_{1} \omega-\sum_{k=1}^{q} \ln \left(1-\gamma_{3 k}\right)=: B_{15}, \\
u_{3}(t) & =u_{3}\left(\eta_{3}\right)-\int_{t}^{\eta_{3}} \dot{u}_{3}(t) d t \geq u_{3}\left(\eta_{3}\right)-\int_{0}^{\omega}\left|\dot{u}_{3}(t)\right| d t \\
& >B_{14}-2 \bar{d}_{1} \omega-\sum_{k=1}^{q} \ln \left(1-\gamma_{3 k}\right)=: B_{16} .
\end{aligned}
$$

Hence, we have

$$
\left|u_{3}(t)\right| \leq \max \left\{\left|B_{15}\right|,\left|B_{16}\right|\right\}=: B_{17} .
$$


Case 3 (if $u_{1}(t)<0, u_{2}(t)>0$ ). By the first equation of (3.14), we have

$$
\begin{gathered}
\int_{0}^{\omega} b_{1}(t) e^{-u_{1}(t)} d t+\sum_{k=1}^{q} \ln \left(1-\gamma_{1 k}\right)<\int_{0}^{\omega} a_{1}(t) d t, \\
\int_{0}^{\omega}\left[b_{1}(t) e^{\left(u_{2}\left(\eta_{2}\right)-u_{1}(t)\right)}\right] d t+\sum_{k=1}^{q} \ln \left(1-\gamma_{1 k}\right)>\int_{0}^{\omega} a_{1}(t) d t .
\end{gathered}
$$

Then

$$
\begin{gathered}
\bar{b}_{1} \omega e^{-u_{1}\left(\eta_{1}\right)}+\sum_{k=1}^{q} \ln \left(1-\gamma_{1 k}\right)<\bar{a}_{1} \omega, \\
\bar{b}_{1} \omega e^{-B_{19}} e^{u_{2}\left(\eta_{2}\right)}+\sum_{k=1}^{q} \ln \left(1-\gamma_{1 k}\right)>\bar{a}_{1} \omega .
\end{gathered}
$$

namely,

$$
\begin{gathered}
u_{1}\left(\eta_{1}\right)>\ln \left[\frac{\bar{b}_{1} \omega}{\bar{a}_{1}-\sum_{k=1}^{q} \ln \left(1-\gamma_{1 k}\right)}\right]=: B_{18}, \\
u_{2}\left(\eta_{2}\right)>\ln \left[\frac{\bar{a}_{1} \omega-\sum_{k=1}^{q} \ln \left(1-\gamma_{1 k}\right)}{\bar{b}_{1} \omega e^{-B_{19}}}\right]=: B_{20} .
\end{gathered}
$$

Therefore,

$$
\begin{aligned}
u_{1}(t) & =u_{1}\left(\eta_{1}\right)-\int_{t}^{\eta_{1}} \dot{u}_{1}(t) d t \geq u_{1}\left(\eta_{1}\right)-\int_{0}^{\omega}\left|\dot{u}_{1}(t)\right| d t \\
& >B_{18}-2 \bar{a}_{1} \omega-\sum_{k=1}^{q} \ln \left(1-\gamma_{1 k}\right)=: B_{19}, \\
u_{2}(t) & =u_{2}\left(\xi_{2}\right)+\int_{\xi_{2}}^{t} \dot{u}_{2}(t) d t \leq u_{2}\left(\xi_{2}\right)+\int_{0}^{\omega}\left|\dot{u}_{2}(t)\right| d t \\
& <\ln \left[\frac{\left|a_{2}-b_{2}\right| \omega+\sum_{k=1}^{q} \ln \left(1-\gamma_{2 k}\right)}{\bar{c} \omega}\right]+2 \bar{a}_{2} \omega+\sum_{k=1}^{q} \ln \left(1-\gamma_{2 k}\right)=: B_{21}, \\
u_{2}(t) & =u_{2}\left(\eta_{2}\right)-\int_{t}^{\eta_{2}} \dot{u}_{2}(t) d t \geq u_{2}\left(\eta_{1}\right)-\int_{0}^{\omega}\left|\dot{u}_{2}(t)\right| d t \\
& >B_{20}-2 \bar{a}_{2} \omega-\sum_{k=1}^{q} \ln \left(1-\gamma_{2 k}\right)=: B_{22} .
\end{aligned}
$$


International Journal of Differential Equations

So

$$
B_{19}<u_{1}(t)<0,\left|u_{2}(t)\right| \leq \max \left\{\left|B_{21}\right|,\left|B_{22}\right|\right\}=: B_{23} .
$$

By the third equation of (3.14), we obtain

$$
\int_{0}^{\omega} e(t) e^{u_{3}\left(\eta_{3}\right)} d t+\int_{0}^{\omega} \beta_{2}(t) e^{B_{23}} d t>\overline{\left|a_{2}-b_{2}\right|} \omega+\sum_{k=1}^{q} \ln \left(1-\gamma_{2 k}\right),
$$

that is,

$$
u_{3}\left(\eta_{3}\right)>\ln \left[\frac{\overline{\left|a_{2}-b_{2}\right|} \omega+\sum_{k=1}^{q} \ln \left(1-\gamma_{2 k}\right)-\bar{\beta}_{2} \omega e^{B_{23}}}{\bar{e} \omega}\right]=: B_{24}
$$

Thus,

$$
\left|u_{3}(t)\right| \leq \max \left\{\left|B_{23}\right|,\left|B_{24}\right|\right\}=: B_{25} .
$$

Case 4 (if $u_{1}(t)<0, u_{2}(t)<0$ ). By the second equation of (3.14), we have

$$
\int_{0}^{\omega} c(t) d t+\int_{0}^{\omega} \beta_{1}(t) e^{u_{3}(t)} d t>\overline{\left|a_{2}-b_{2}\right|} \omega+\sum_{k=1}^{q} \ln \left(1-\gamma_{2 k}\right) .
$$

Then

$$
\bar{c} \omega+\bar{\beta}_{1} \omega e^{u_{3}\left(\eta_{3}\right)}>\overline{\left|a_{2}-b_{2}\right|} \omega+\sum_{k=1}^{q} \ln \left(1-\gamma_{2 k}\right),
$$

that is,

$$
u_{3}\left(\eta_{3}\right) \geq \ln \left[\frac{\overline{\left|a_{2}-b_{2}\right|} \omega+\sum_{k=1}^{q} \ln \left(1-\gamma_{2 k}\right)-\bar{c} \omega}{\bar{\beta}_{1} \omega}\right]=: B_{26} .
$$

Therefore,

$$
\begin{aligned}
u_{3}(t) & =u_{3}\left(\xi_{3}\right)+\int_{\xi_{3}}^{t} \dot{u}_{3}(t) d t \leq u_{3}\left(\xi_{3}\right)+\int_{0}^{\omega}\left|\dot{u}_{3}(t)\right| d t \\
& <\ln \left[\frac{\bar{d} \omega+\sum_{k=1}^{q} \ln \left(1-\gamma_{3 k}\right)}{\bar{e} \omega}\right]+2 \bar{d} \omega+\sum_{k=1}^{q} \ln \left(1-\gamma_{3 k}\right)=: B_{27}
\end{aligned}
$$




$$
\begin{aligned}
u_{3}(t) & =u_{3}\left(\eta_{3}\right)-\int_{t}^{\eta_{3}} \dot{u}_{3}(t) d t \geq u_{3}\left(\eta_{3}\right)-\int_{0}^{\omega}\left|\dot{u}_{3}(t)\right| d t \\
& >B_{26}-2 \bar{d} \omega-\sum_{k=1}^{q} \ln \left(1-\gamma_{3 k}\right)=: B_{28} .
\end{aligned}
$$

Thus,

$$
\left|u_{3}(t)\right| \leq \max \left\{\left|B_{27}\right|,\left|B_{28}\right|\right\}=: B_{29}
$$

By the second equation of (3.14), we obtain

$$
\int_{0}^{\omega} c(t) e^{u_{2}\left(\eta_{2}\right)} d t+\int_{0}^{\omega} \beta_{1}(t) e^{B_{29}} d t>\overline{\left|a_{2}-b_{2}\right|} \omega+\sum_{k=1}^{q} \ln \left(1-\gamma_{2 k}\right)
$$

that is,

$$
\bar{c} e^{u_{2}\left(\eta_{2}\right)}+\bar{\beta}_{1} \omega e^{B_{29}}>\overline{\left|a_{2}-b_{2}\right|} \omega+\sum_{k=1}^{q} \ln \left(1-\gamma_{2 k}\right) .
$$

Thus,

$$
u_{2}\left(\eta_{2}\right)>\ln \left[\frac{\overline{\left|a_{2}-b_{2}\right|} \omega+\sum_{k=1}^{q} \ln \left(1-\gamma_{2 k}\right)-\bar{\beta}_{1} \omega e^{B_{29}}}{\bar{c} \omega}\right] .
$$

Then, from (3.16) and (3.20), we get

$$
\begin{aligned}
u_{2}(t) & =u_{2}\left(\xi_{2}\right)+\int_{\xi_{2}}^{t} \dot{u}_{2}(t) d t \leq u_{2}\left(\xi_{2}\right)+\int_{0}^{\omega}\left|\dot{u}_{2}(t)\right| d t \\
& <\ln \left[\frac{\overline{\left|a_{2}-b_{2}\right|} \omega+\sum_{k=1}^{q} \ln \left(1-\gamma_{2 k}\right)}{\bar{c} \omega}\right]+2 \bar{a}_{2} \omega+\sum_{k=1}^{q} \ln \left(1-\gamma_{2 k}\right)=: B_{31}, \\
u_{2}(t) & =u_{2}\left(\eta_{2}\right)-\int_{t}^{\eta_{2}} \dot{u}_{2}(t) d t \geq u_{2}\left(\eta_{2}\right)-\int_{0}^{\omega}\left|\dot{u}_{2}(t)\right| d t \\
& >B_{30}-2 \bar{a}_{2} \omega \omega-\sum_{k=1}^{q} \ln \left(1-\gamma_{2 k}\right)=: B_{32} .
\end{aligned}
$$

Thus,

$$
\left|u_{2}(t)\right| \leq \max \left\{\left|B_{31}\right|,\left|B_{32}\right|\right\}=: B_{33} .
$$


By the first equation of (3.14), we have

$$
\int_{0}^{\omega} b_{1}(t) e^{u_{2}(t)-u_{1}\left(\eta_{1}\right)} d t+\sum_{k=1}^{q} \ln \left(1-\gamma_{1 k}\right)<\int_{0}^{\omega} a_{1}(t) d t
$$

Then

$$
\bar{b}_{1} \omega e^{-B_{33}} e^{-u_{1}\left(\eta_{1}\right)}+\sum_{k=1}^{q} \ln \left(1-\gamma_{1 k}\right)<\bar{a}_{1} \omega
$$

Thus,

$$
u_{1}\left(\eta_{1}\right)>\ln \left[\frac{\bar{b}_{1} \omega e^{-B_{32}}}{\bar{a}_{1} \omega-\sum_{k=1}^{q} \ln \left(1-\gamma_{1 k}\right)}\right]=: B_{34} .
$$

Hence, we have

$$
B_{34}<u_{1}(t)<0
$$

Based on the discussion above, we can easily obtain

$$
\begin{gathered}
u_{1}(t) \leq \max \left\{B_{5}, B_{12},\left|B_{19}\right|,\left|B_{34}\right|\right\}, \\
u_{2}(t) \leq \max \left\{B_{3}, B_{4}, B_{23}, B_{33}\right\}, \\
u_{3}(t) \leq \max \left\{B_{8}, B_{17}, B_{25}, B_{29}\right\} .
\end{gathered}
$$

Obviously, $B_{i}(i=1,2, \ldots, 34)$ are independent of $\lambda \in(0,1)$. Similar to the proof of Theorem 2.1 of [17], we can easily find a sufficiently large $M>0$ so that we denote the set

$$
\Omega=\left\{u(t)=\left(u_{1}(t), u_{2}(t), u_{3}(t)\right)^{T} \in x:\|u\|<M, u\left(t_{k}^{+}\right) \in \Omega, k=1,2, \ldots, q\right\} .
$$

It is clear that $\Omega$ satisfies the requirement (a) in Lemma 3.1.

When $\left(u_{1}(t), u_{2}(t), u_{3}(t)\right)^{T} \in \partial \Omega \cap \operatorname{Ker} L=\partial \Omega \cap R^{3}$ and $u=\left\{\left(u_{1}, u_{2}, u_{3}\right)^{T}\right\}$ is a constant vector in $R^{3}$ with $\|u\|=\left\|\left(u_{1}(t), u_{2}(t), u_{3}(t)\right)^{T}\right\|=M$, then we have

$$
Q N u=\left(\left(\begin{array}{c}
\bar{a}_{1}+\bar{b}_{1} e^{u_{2}-u_{1}}+\frac{1}{\omega} \sum_{k=1}^{q} \ln \left(1-\gamma_{1 k}\right) \\
\bar{a}_{2}-b_{2}-\bar{c} e^{u_{2}} \bar{\beta}_{1} e^{u_{3}}+\frac{1}{\omega} \sum_{k=1}^{q} \ln \left(1-\gamma_{2 k}\right) \\
\bar{d}-\bar{e} e^{u_{3}}-\bar{\beta}_{2} e^{u_{2}}+\frac{1}{\omega} \sum_{k=1}^{q} \ln \left(1-\gamma_{3 k}\right)
\end{array}\right), 0, \ldots, 0\right) \neq 0 .
$$


Letting $J: \operatorname{Im} Q \rightarrow \operatorname{Ker} L,(r, 0, \ldots, 0,0) \rightarrow r$ and, by direct calculation, we get

$$
\begin{aligned}
\operatorname{deg}\left\{\operatorname{JQN}\left(u_{1}, u_{2}, u_{3}\right)^{T} ; \partial \Omega \bigcap \operatorname{ker} L ; 0\right\} \\
=\operatorname{signdet}\left(\begin{array}{ccc}
-\bar{b}_{1} e^{u_{2}-u_{1}} & \bar{b}_{1} e^{u_{2}-u_{1}} & 0 \\
0 & -\bar{c} e^{u_{2}} & -\bar{\beta}_{1} e^{u_{3}} \\
0 & -\bar{\beta}_{2} e^{u_{2}} & -\bar{e} e^{u_{3}}
\end{array}\right) \\
=\operatorname{sign}\left\{\left(\bar{b}_{1} \bar{\beta}_{1} \bar{\beta}_{2}-\bar{b}_{1} \overline{c e}\right) e^{2 u_{2}-u_{1}+u_{3}}\right\} \neq 0 .
\end{aligned}
$$

This proves that condition (b) in Lemma 3.1 is satisfied. By now, we have proved that $\Omega$ verifies all requirements of Lemma 3.1, then it follows that $L u=N u$ has at least one solution $\left(u_{1}(t), u_{2}(t), u_{3}(t)\right)^{T}$ in Dom $L \cap \bar{\Omega}$; that is, to say, (3.5) has at least one $\omega$ periodic solution in Dom $L \cap \bar{\Omega}$. Then we know that $\left(\left(x_{1}(t), x_{2}(t), x_{3}(t)\right)^{T}=\left(e^{u_{1}(t)}, e x^{u_{2}(t)}, e^{u_{3}(t)}\right)^{T}\right.$ is an $\omega$ periodic solution of system (2.4) with strictly positive components. This completes the proof.

\section{Uniqueness and Global Attractivity of Periodic Solutions}

Under the hypotheses $\left(\mathrm{H}_{1}\right),\left(\mathrm{H}_{2}\right),\left(\mathrm{H}_{3}\right)$, we consider the following ordinary differential equation without impulsive:

$$
\begin{gathered}
\dot{z}_{1}(t)=z_{1}(t)\left[-a_{1}(t)+b_{1}(t) \frac{\prod_{0<t_{k}<t}\left(1-\gamma_{2 k}\right) z_{2}(t)}{\prod_{0<t_{k}<t}\left(1-\gamma_{1 k}\right) z_{1}(t)}\right], \\
\dot{z}_{2}(t)=z_{2}(t)\left[a_{2}(t)-b_{2}(t)-c(t) \prod_{0<t_{k}<t}\left(1-\gamma_{2 k}\right) z_{2}(t)-\beta_{1}(t) \prod_{0<t_{k}<t}\left(1-\gamma_{3 k}\right) z_{3}(t)\right], \\
\dot{z}_{3}(t)=z_{3}(t)\left[d(t)-e(t) \prod_{0<t_{k}<t}\left(1-\gamma_{3 k}\right) z_{3}(t)-\beta_{2}(t) \prod_{0<t_{k}<t}\left(1-\gamma_{2 k}\right) z_{2}(t)\right],
\end{gathered}
$$

with the initial conditions $z_{i}(0)>0, i=1,2,3$.

The following lemmas will be helpful in the proofs of our results. The proof of the following Lemma 4.1 is similar to that of Theorem 1 in [18], and it will be omitted.

Lemma 4.1. Assume that $\left(\mathrm{H}_{1}\right),\left(\mathrm{H}_{2}\right),\left(\mathrm{H}_{3}\right)$ hold, then one has the following.

(i) If $z(t)=\left(z_{1}(t), z_{2}(t), z_{3}(t)\right)^{T}$ is a solution of $(4.1)$ on $[0,+\infty)$, then $x_{i}(t)=\prod_{0<t_{k}<t}(1-$ $\left.\gamma_{i k}\right) z_{i}(t)(i=1,2,3)$ is a solution of $(2.4)$ on $[0,+\infty)$.

(ii) If $x(t)=\left(x_{1}(t), x_{2}(t), x_{3}(t)\right)^{T}$ is a solution of $(2.4)$ on $[0,+\infty)$, then $z_{i}(t)=$ $\prod_{0<t_{k}<t}\left(1-\gamma_{i k}\right)^{-1} x_{i}(t)(i=1,2,3)$ is a solution of $(4.1)$ on $[0,+\infty)$.

Lemma 4.2. Let $z(t)=\left(z_{1}(t), z_{2}(t), z_{3}(t)\right)^{T}$ denote any positive solution of system (4.1) with initial conditions $z_{i}(0)>0, i=1,2,3$. Assume that the following condition holds,

$$
\left(\mathrm{H}_{7}\right) a_{2}^{M}>b_{2}^{L}, \quad d^{M}>e^{L} .
$$


Then there exists a $T_{3}>0$ such that

$$
0<z_{i}(t) \leq M_{i}, \quad(i=1,2,3), \text { for } t \geq T_{3},
$$

where

$$
\begin{aligned}
& M_{1}>M_{1}^{*}=\frac{\prod_{0<t_{k}<t}\left(1-\gamma_{2 k}\right) M_{2}}{a_{1}^{L} \prod_{0<t_{k}<t}\left(1-\gamma_{1 k}\right)}, \\
& M_{2}>M_{2}^{*}=\frac{a_{2}^{M}-b_{2}^{L}}{c^{L} \prod_{0<t_{k}<t}\left(1-\gamma_{2 k}\right)}, \\
& M_{3}>M_{3}^{*}=\frac{d^{M}-e^{L}}{e^{L} \prod_{0<t_{k}<t}\left(1-\gamma_{3 k}\right)} .
\end{aligned}
$$

Proof. From the second equation of (4.1), we can obtain

$$
\begin{aligned}
\dot{z}_{2}(t) & \leq z_{2}(t)\left[a_{2}(t)-b_{2}(t)-c(t) \prod_{0<t_{k}<t}\left(1-\gamma_{2 k}\right) z_{2}(t)\right] \\
& \leq z_{2}(t)\left[a_{2}^{M}-b_{2}^{L}-c^{L} \prod_{0<t_{k}<t}\left(1-\gamma_{2 k}\right) z_{2}(t)\right] .
\end{aligned}
$$

By (4.5), we can derive the following.

$\left(\mathrm{A}_{1}\right)$ If $z_{2}(0) \leq M_{2}$, then $z_{2}(t) \leq M_{2}, t \geq 0$.

$\left(\mathrm{A}_{2}\right)$ If $z_{2}(0)>M_{2}$, let $-\alpha_{1}=M_{2}\left[a_{2}^{M}-b_{2}^{L}-c^{L} \prod_{0<t_{k}<t}\left(1-\gamma_{2 k}\right) M_{2}\right], \quad\left(\alpha_{1}>0\right)$. Then there exists $\varepsilon_{1}>0$ such that $t \in\left[0, \varepsilon_{1}\right)$, then $z_{2}(t)>M_{2}$, and also we have

$$
\dot{z}_{2}(t)<-\alpha_{1}<0 \text {. }
$$

From what has been discussed above, we can easily conclude that, if $z_{2}(0)>M_{2}$, then $z_{2}(t)$ is strictly monotone decreasing with speed at least $\alpha_{1}$. Therefore, there exists a $T_{1}>0$ such that $t>T_{1}$, then $z_{2}(t) \leq M_{2}$.

From the third equation of (4.1), we can obtain

$$
\begin{aligned}
\dot{z}_{3}(t) & \leq z_{3}(t)\left[d(t)-e(t) \prod_{0<t_{k}<t}\left(1-\gamma_{3 k}\right) z_{3}(t)\right] \\
& \leq z_{3}(t)\left[d^{M}-e^{L} \prod_{0<t_{k}<t}\left(1-\gamma_{3 k}\right) z_{3}(t)\right] .
\end{aligned}
$$

By (4.7), we can derive the following

$\left(\mathrm{B}_{1}\right)$ If $z_{3}(0) \leq M_{3}$, then $z_{3}(t) \leq M_{3}, t \geq 0$. 
$\left(B_{2}\right)$ If $z_{3}(0)>M_{3}$, let $-\alpha_{2}=M_{3}\left[d^{M}-e^{L} \prod_{0<t_{k}<t}\left(1-\gamma_{3 k}\right) M_{3}\right],\left(\alpha_{2}>0\right)$. Then there exists $\varepsilon_{2}>0$ such that $t \in\left[0, \varepsilon_{2}\right)$, then $z_{3}(t)>M_{3}$, and also we have

$$
\dot{z}_{3}(t)<-\alpha_{2}<0 \text {. }
$$

From what has been discussed above, we can easily conclude that, if $z_{3}(0)>M_{3}$, then $z_{3}(t)$ is strictly monotone decreasing with speed at least $\alpha_{2}$. Therefore, there exists a $T_{2}>0$ such that $t>T_{2}$, then $z_{3}(t) \leq M_{3}$.

From the first equation of (4.1), we can obtain

$$
\begin{aligned}
\dot{z}_{1}(t) & \leq z_{1}(t)\left[-a_{1}(t)+b_{1}(t) \frac{\prod_{0<t_{k}<t}\left(1-\gamma_{2 k}\right) M_{2}}{\prod_{0<t_{k}<t}\left(1-\gamma_{1 k}\right) z_{1}(t)}\right] \\
& =-a_{1}(t) z_{1}(t)+\frac{\prod_{0<t_{k}<t}\left(1-\gamma_{2 k}\right) M_{2}}{\prod_{0<t_{k}<t}\left(1-\gamma_{1 k}\right)} \\
& \leq-a_{1}^{L} z_{1}(t)+\frac{\prod_{0<t_{k}<t}\left(1-\gamma_{2 k}\right) M_{2}}{\prod_{0<t_{k}<t}\left(1-\gamma_{1 k}\right)} .
\end{aligned}
$$

Then we have

$$
z_{1}(t) \leq M_{1}, \quad \text { for } t \geq T_{1}
$$

Set $T_{3}=\max \left\{T_{1}, T_{2}\right\}$, then we have

$$
0<z_{i}(t) \leq M_{i}, \quad(i=1,2,3), \quad \text { for } t \geq T_{3} .
$$

The proof is complete.

Lemma 4.3. Let $\left(\mathrm{H}_{1}\right),\left(\mathrm{H}_{2}\right),\left(\mathrm{H}_{3}\right)$ hold. Assume that the following condition holds.

$$
\left(\mathrm{H}_{8}\right) a_{2}^{L}>b_{2}^{M}+\beta_{1}^{M} \prod_{0<t_{k}<t}\left(1-\gamma_{3 k}\right), \quad d^{L}>e^{M}-\beta_{2}^{M} \prod_{0<t_{k}<t}\left(1-\gamma_{2 k}\right) .
$$

Then there exists positive constants $T>0$ and $m_{i}(i=1,2,3)$ such that, for all $t>T$,

$$
m_{i}<z_{i}(t), \quad(i=1,2,3), \quad \text { for } t \geq T,
$$

in which

$$
m_{1}<m_{1}^{*}=\frac{b_{1}^{L} \prod_{0<t_{k}<t}\left(1-\gamma_{2 k}\right) m_{2}}{a_{1}^{M} \prod_{0<t_{k}<t}\left(1-\gamma_{1 k}\right)},
$$


International Journal of Differential Equations

$$
\begin{aligned}
& m_{2}<m_{2}^{*}=\frac{a_{2}^{L}-b_{2}^{M}-\beta_{1}^{M} \prod_{0<t_{k}<t}\left(1-\gamma_{3 k}\right)}{c^{M} \prod_{0<t_{k}<t}\left(1-\gamma_{2 k}\right)}, \\
& m_{3}<m_{3}^{*}=\frac{d^{L}-e^{M}-\beta_{2}^{M} \prod_{0<t_{k}<t}\left(1-\gamma_{2 k}\right) M_{2}}{e^{M} \prod_{0<t_{k}<t}\left(1-\gamma_{3 k}\right)} .
\end{aligned}
$$

Proof. By the second equation of (4.1), It is easy to obtain that, for $t \geq T_{3}$,

$$
\dot{z}_{2}(t) \geq z_{2}(t)\left[a_{2}^{L}-b_{2}^{M}-c^{M} \prod_{0<t_{k}<t}\left(1-\gamma_{2 k}\right) z_{2}(t)-\beta_{1}^{M} \prod_{0<t_{k}<t}\left(1-\gamma_{3 k}\right) M_{3}\right],
$$

where $T_{3}$ is defined in Lemma 4.1.

$\left(C_{1}\right)$ If $z_{2}\left(T_{3}\right) \geq m_{2}$, then $z_{2}(t) \geq m_{2}, t \geq T_{3}$.

$\left(C_{2}\right)$ If $z_{2}\left(T_{3}\right)<m_{2}$ and let

$$
\mu_{1}=z_{2}\left(T_{3}\right)\left[a_{2}^{L}-b_{2}^{M}-c^{M} \prod_{0<t_{k}<t}\left(1-\gamma_{2 k}\right) m_{2}\right]
$$

then there exists $\varepsilon_{3}>0$ such that $t \in\left[T_{3}, T_{3}+\varepsilon_{3}\right)$, then $z_{2}(t)>m_{2}$, and also we have

$$
\dot{z}_{2}(t)>\mu_{1}>0
$$

Then we know that if $z_{2}\left(T_{3}\right)<m_{2}, z_{2}(t)$ will strictly monotonically increase with speed $\mu_{2}$. Thus, there exists $T_{4}>T_{3}$ such that if $t \geq T_{4}$, then $z_{2}(t) \geq m_{2}$.

By the third equation of (4.1), It is easy to obtain that for $t \geq T_{3}$,

$$
\dot{z}_{3}(t) \geq z_{3}(t)\left[d^{L}-e^{M} \prod_{0<t_{k}<t}\left(1-\gamma_{3 k}\right) z_{3}(t)-\beta_{2}^{M} \prod_{0<t_{k}<t}\left(1-\gamma_{2 k}\right) M_{2}\right],
$$

where $T_{3}$ is defined in Lemma 4.2 .

$\left(\mathrm{D}_{1}\right)$ If $z_{2}\left(T_{3}\right) \geq m_{3}$, then $z_{3}(t) \geq m_{3}, t \geq T_{3}$.

$\left(\mathrm{D}_{2}\right)$ If $z_{2}\left(T_{3}\right)<m_{3}$, and let

$$
\mu_{2}=z_{3}\left(T_{3}\right)\left[d^{L}-e^{M} \prod_{0<t_{k}<t}\left(1-\gamma_{3 k}\right) m_{3}-\beta_{2}^{M} \prod_{0<t_{k}<t}\left(1-\gamma_{2 k}\right) M_{2}\right],
$$

then there exists $\varepsilon_{4}>0$ such that $t \in\left[T_{3}, T_{3}+\varepsilon_{4}\right)$, then $z_{3}(t)>m_{3}$, and also we have

$$
\dot{z}_{3}(t)>\mu_{2}>0
$$


Then we know that if $z_{3}\left(T_{3}\right)<m_{3}, z_{3}(t)$ will strictly monotonically increase with speed $\mu_{2}$. Thus, there exists $T_{5}>T_{3}$ such that, if $t \geq T_{5}$, then $z_{3}(t) \geq m_{3}$.

Finally, by the third equation of (4.1), we obtain

$$
\begin{aligned}
\dot{z}_{1}(t) & \geq z_{1}(t)\left[-a_{1}^{M}+b_{1}^{L} \frac{\prod_{0<t_{k}<t}\left(1-\gamma_{2 k}\right) m_{2}}{\prod_{0<t_{k}<t}\left(1-\gamma_{1 k}\right) z_{1}(t)}\right] \\
& =-a_{1}^{M} z_{1}(t)+b_{1}^{L} \frac{\prod_{0<t_{k}<t}\left(1-\gamma_{2 k}\right) m_{2}}{\prod_{0<t_{k}<t}\left(1-\gamma_{1 k}\right) z_{1}(t)} .
\end{aligned}
$$

Thus, we have

$$
z_{1}(t) \geq m_{1}
$$

for $t \geq T_{4}$. Set $T=\max \left\{T_{4}, T_{5}\right\}$, then we have

$$
z_{i}(t)>m_{i}, \quad(i=1,2,3), \quad \text { for } t \geq T .
$$

In the sequel, we formulate the uniqueness and global attractivity of the $\omega$ periodic solution $x^{*}(t)$ in Theorem 4.4. It is immediate that if $x^{*}(t)$ is global attractivity, then $x^{*}(t)$ is in fact unique.

Theorem 4.4. In addition to $\left(\mathrm{H}_{1}\right)-\left(\mathrm{H}_{8}\right)$, assume further $\left(\mathrm{H}_{9}\right) \lim _{t \rightarrow \infty}$ inf $B_{i}(t)>0$, where

$$
\begin{aligned}
& B_{1}(t)=\left[c(t)-\beta_{2}(t)-\frac{b^{M}}{m_{1} \prod_{0<t_{k}<t}\left(1-\gamma_{1 k}\right)}\right] \prod_{0<t_{k}<t}\left(1-\gamma_{2 k}\right), \\
& B_{2}(t)=\left[e(t)-\beta_{1}(t)\right] \prod_{0<t_{k}<t}\left(1-\gamma_{3 k}\right) .
\end{aligned}
$$

Then system (2.4) has a unique positive $\omega$ periodic solution $x^{*}(t)=\left(x_{1}^{*}(t), x_{2}^{*}(t), x_{3}^{*}(t)\right)^{T}$ which is global attractivity.

Proof. According to the conclusion of Theorem 3.2, we only need to show that the positive periodic solution of (2.4) is global asymptotical stable. Let $x^{*}(t)=\left(x_{1}^{*}(t), x_{2}^{*}(t), x_{3}^{*}(t)\right)^{T}$ be a positive $\omega$ periodic solution of system (2.4) let $x(t)=\left(x_{1}(t), x_{2}(t), x_{3}(t)\right)^{T}$ be any positive solution of system $(2.4)$. Then $z^{*}(t)=\left(z_{1}^{*}(t), z_{2}^{*}(t), z_{3}^{*}(t)\right)^{T},\left(z_{1}^{*}(t)=\prod_{0<t_{k}<t}(1-\right.$ $\left.\left.\gamma_{1 k}\right) x_{1}^{*}(t), z_{2}^{*}(t)=\prod_{0<t_{k}<t}\left(1-\gamma_{2 k}\right) x_{2}^{*}(t), z_{3}^{*}(t)=\prod_{0<t_{k}<t}\left(1-\gamma_{3 k}\right) x_{3}^{*}(t)\right)$ is the positive $\omega$ periodic solution of (4.1), and $z(t)$ is the positive solution of (4.1). It follows from Lemma 4.2 and 4.3 that there exists positive constants $T>0, M_{i}$ and $m_{i}$ (defined by Lemmas 4.2 and 4.3, resp.) such that, for all $t>T$,

$$
m_{i}<z_{i}^{*}(t) \leq M_{i}, \quad m_{i}<z_{i}(t) \leq M_{i}, i=1,2,3 .
$$


International Journal of Differential Equations

Define

$$
V(t)=\left|\ln z_{1}^{*}(t)-\ln z_{1}(t)\right|+\left|\ln z_{2}^{*}(t)-\ln z_{2}(t)\right|+\left|\ln z_{3}^{*}(t)-\ln z_{3}(t)\right|
$$

Calculating the upper-right derivative of $V(t)$ along the solution of (4.1), it follows for $t \geq$ $T$ that

$$
\begin{aligned}
D^{+} V(t)= & \sum_{i=1}^{3}\left(\frac{z_{i}^{* \prime}(t)}{z_{i}^{*}(t)}-\frac{z_{i}^{\prime}(t)}{z_{i}(t)}\right) \operatorname{sgn}\left(z_{i}^{*}(t)-z_{i}(t)\right) \\
= & \operatorname{sgn}\left(z_{1}^{*}(t)-z_{1}(t)\right)\left[b_{1}(t) \frac{\prod_{0<t_{k}<t}\left(1-\gamma_{2 k}\right)}{\prod_{0<t_{k}<t}\left(1-\gamma_{1 k}\right)}\left(\frac{z_{2}^{*}(t)}{z_{1}^{*}(t)}-\frac{z_{2}(t)}{z_{1}(t)}\right)\right] \\
& +\operatorname{sgn}\left(z_{2}^{*}(t)-z_{2}(t)\right)\left[-c(t) \prod_{0<t_{k}<t}\left(1-\gamma_{2 k}\right)\left(z_{2}^{*}(t)-z_{2}(t)\right)\right. \\
& +\operatorname{sgn}\left(z_{3}^{*}(t)-z_{3}(t)\right)\left[-e(t) \prod_{0<t_{k}<t}\left(1-\gamma_{3 k}\right)\left(z_{3}^{*}(t)-z_{3}(t)\right)\right. \\
\left.\quad-\beta_{2}(t) \prod_{0<t_{k}<t}\left(1-\gamma_{2 k}\right)\left(z_{2}^{*}(t)-z_{2}(t)\right)\right] & \left.\left(z_{3}^{*}(t)-z_{3}(t)\right)\right] \\
\leq & -c(t) \prod_{0<t_{k}<t}\left(1-\gamma_{2 k}\right)\left|z_{2}^{*}(t)-z_{2}(t)\right|+\beta_{1}(t) \prod_{0<t_{k}<t}\left(1-\gamma_{3 k}\right)\left|z_{3}^{*}(t)-z_{3}(t)\right| \\
& -e(t) \prod_{0<t_{k}<t}\left(1-\gamma_{3 k}\right)\left|z_{3}^{*}(t)-z_{3}(t)\right|+\beta_{2}(t) \prod_{0<t_{k}<t}\left(1-\gamma_{2 k}\right)\left|z_{2}^{*}(t)-z_{2}(t)\right|+D_{1}(t),
\end{aligned}
$$

where

$$
D_{1}(t)= \begin{cases}b_{1}(t) \frac{\prod_{0<t_{k}<t}\left(1-\gamma_{2 k}\right)}{\prod_{0<t_{k}<t}\left(1-\gamma_{1 k}\right)}\left(\frac{z_{2}^{*}(t)}{z_{1}^{*}(t)}-\frac{z_{2}(t)}{z_{1}(t)}\right), & z_{1}^{*}(t)>z_{1}(t), \\ b_{1}(t) \frac{\prod_{0<t_{k}<t}\left(1-\gamma_{2 k}\right)}{\prod_{0<t_{k}<t}\left(1-\gamma_{1 k}\right)}\left(\frac{z_{2}^{*}(t)}{z_{1}^{*}(t)}-\frac{z_{2}(t)}{z_{1}(t)}\right), & z_{1}^{*}(t)<z_{1}(t) .\end{cases}
$$

In the sequel, we will estimate $D_{1}(t)$ under the following two cases.

(i) If $z_{1}^{*}(t) \geq z_{1}(t)$, then

$$
\begin{aligned}
D_{1}(t) & \leq \frac{b_{1}(t) \prod_{0<t_{k}<t}\left(1-\gamma_{2 k}\right)}{z_{1}^{*}(t) \prod_{0<t_{k}<t}\left(1-\gamma_{1 k}\right)}\left(z_{2}^{*}(t)-z_{2}(t)\right) \\
& \leq \frac{b^{M} \prod_{0<t_{k}<t}\left(1-\gamma_{2 k}\right)}{m_{1} \prod_{0<t_{k}<t}\left(1-\gamma_{1 k}\right)}\left|z_{2}^{*}(t)-z_{2}(t)\right| .
\end{aligned}
$$


(ii) If $z_{1}^{*}(t)<z_{1}(t)$, then

$$
\begin{aligned}
D_{1}(t) & \leq \frac{b_{1}(t) \prod_{0<t_{k}<t}\left(1-\gamma_{2 k}\right)}{z_{1}(t) \prod_{0<t_{k}<t}\left(1-\gamma_{1 k}\right)}\left(z_{2}(t)-z_{2}^{*}(t)\right) \\
& \leq \frac{b^{M} \prod_{0<t_{k}<t}\left(1-\gamma_{2 k}\right)}{m_{1} \prod_{0<t_{k}<t}\left(1-\gamma_{1 k}\right)}\left|z_{2}^{*}(t)-z_{2}(t)\right| .
\end{aligned}
$$

Combining the conclusions of (4.29) and (4.30), we obtain

$$
D_{1}(t) \leq \frac{b^{M} \prod_{0<t_{k}<t}\left(1-\gamma_{2 k}\right)}{m_{1} \prod_{0<t_{k}<t}\left(1-\gamma_{1 k}\right)}\left|z_{2}^{*}(t)-z_{2}(t)\right| .
$$

It follows from (4.31) that

$$
\begin{aligned}
D^{+} V(t) \leq & -c(t) \prod_{0<t_{k}<t}\left(1-\gamma_{2 k}\right)\left|z_{2}^{*}(t)-z_{2}(t)\right|+\beta_{1}(t) \prod_{0<t_{k}<t}\left(1-\gamma_{3 k}\right)\left|z_{3}^{*}(t)-z_{3}(t)\right| \\
& -e(t) \prod_{0<t_{k}<t}\left(1-\gamma_{3 k}\right)\left|z_{3}^{*}(t)-z_{3}(t)\right|+\beta_{2}(t) \prod_{0<t_{k}<t}\left(1-\gamma_{2 k}\right)\left|z_{2}^{*}(t)-z_{2}(t)\right| \\
& +\frac{b^{M} \prod_{0<t_{k}<t}\left(1-\gamma_{2 k}\right)}{m_{1} \prod_{0<t_{k}<t}\left(1-\gamma_{1 k}\right)}\left|z_{2}^{*}(t)-z_{2}(t)\right| \\
= & {\left[\frac{b^{M}}{m_{1} \prod_{0<t_{k}<t}\left(1-\gamma_{1 k}\right)}-c(t)+\beta_{2}(t)\right] \prod_{0<t_{k}<t}\left(1-\gamma_{2 k}\right)\left|z_{2}^{*}(t)-z_{2}(t)\right| } \\
& +\left[\beta_{1}(t)-e(t)\right] \prod_{0<t_{k}<t}\left(1-\gamma_{3 k}\right)\left|z_{3}^{*}(t)-z_{3}(t)\right| \\
\leq & -\left(B_{1}(t)\left|z_{2}^{*}(t)-z_{2}(t)\right|+B_{2}(t)\left|z_{3}^{*}(t)-z_{3}(t)\right|\right),
\end{aligned}
$$

where $B_{1}(t)$ and $B_{2}(t)$ are defined in Theorem 4.4.

By hypothesis $\left(\mathrm{H}_{8}\right)$, there exist constants $\alpha_{i},(i=2,3)$ and $T^{*}>T$ such that

$$
B_{i}(t) \geq \alpha_{i}>0, \quad(i=2,3), \text { for } t \geq T^{*} .
$$

Integrating both sides of (4.32) on interval $\left[T^{*}, t\right]$ yields

$$
V(t)+\sum_{i=2}^{3} \int_{T^{*}}^{t} B_{i}(t)\left|z_{i}^{*}(t)-z_{i}(t)\right| d s \leq V\left(T^{*}\right)
$$


It follows from (4.33) and (4.34) that

$$
\sum_{i=2}^{3} \int_{T^{*}}^{t} B_{i}(t)\left|z_{i}^{*}(t)-z_{i}(t)\right| d s \leq V\left(T^{*}\right)<\infty, \quad \text { for } t \geq T^{*}
$$

Since $z_{i}^{*}(t)$ and $z_{i}(t)(i=2,3)$ are bounded for $t \geq T^{*}$, so $\left|z_{i}^{*}(t)-z_{i}(t)\right|(i=2,3)$ are uniformly continuous on $\left[T^{*}, \infty\right)$. By Barbalat's Lemma [32], we have

$$
\lim _{t \rightarrow \infty}\left|z_{i}^{*}(t)-z_{i}(t)\right|=\lim _{t \rightarrow \infty}\left[\prod_{0<t_{k}<t}\left(1-\gamma_{i k}\right)^{-1}\left|x_{i}^{*}(t)-x_{i}(t)\right|\right]=0, \quad(i=2,3)
$$

Thus,

$$
\lim _{t \rightarrow \infty}\left|x_{i}^{*}(t)-x_{i}(t)\right|=0, \quad(i=2,3)
$$

By (4.37) and the first equation of (2.4), one can easily obtain that

$$
\lim _{t \rightarrow \infty}\left|x_{1}^{*}(t)-x_{1}(t)\right|=0
$$

By Theorems 7.4 and 8.2 in [33], we know that the positive periodic solution $x^{*}(t)=$ $\left(x_{1}^{*}(t), x_{2}^{*}(t), x_{3}^{*}(t)\right)^{T}$ of $(2.4)$ is uniformly asymptotically stable. The proof of Theorem 4.4 is complete.

\section{An Example}

As an application of our main results, we consider the following system:

$$
\begin{gathered}
\dot{x}_{1}(t)=-2 x_{1}(t)+x_{2}(t), \quad t \neq t_{k}, \\
\dot{x}_{2}(t)=(4+\cos t) x_{2}(t)-(2+\cos t) x_{2}(t)-(1-\sin t) x_{2}^{2}(t) \\
-\frac{1}{2 e^{200 \pi+1}}+\sin t x_{2}(t) x_{3}(t), \quad t \neq t_{k}, \\
\dot{x}_{3}(t)=x_{3}(t)\left[50+\sin t-\left(50 e^{200 \pi}+1+\sin t\right) x_{3}(t)-\left(\frac{49}{e^{50} 3 \pi}-\cos t\right) x_{2}(t)\right], \quad t \neq t_{k}, \\
\Delta x_{1}\left(t_{k}\right)=x_{1}\left(t_{k}^{+}\right)-x_{1}\left(t_{k}^{-}\right)=-\frac{1}{2} x_{1}\left(t_{k}\right), \quad k=1,2, \ldots, \\
\Delta x_{2}\left(t_{k}\right)=x_{2}\left(t_{k}^{+}\right)-x_{2}\left(t_{k}^{-}\right)=-\frac{1}{3} x_{2}\left(t_{k}\right), \quad k=1,2, \ldots, \\
\Delta x_{3}\left(t_{k}\right)=x_{3}\left(t_{k}^{+}\right)-x_{3}\left(t_{k}^{-}\right)=-\frac{1}{4} x_{3}\left(t_{k}\right), \quad k=1,2, \ldots,
\end{gathered}
$$


in which $t_{k+2}=t_{k}+2 \pi,[0,2 \pi] \cap\left\{t_{k}\right\}=\left\{t_{1}, t_{2}\right\}, a_{1}(t)=2, b_{1}(t)=1, a_{2}(t)=4+\cos t, b_{2}(t)=$ $2+\cos t, \beta_{1}(t)=\left(1 / 2 e^{200 \pi+1}\right)+\sin t, \beta_{2}(t)=\left(49 / e^{50} 3 \pi\right)-\cos t, c(t)=1-\sin t, d(t)=50+\sin t$, and $e(t)=50 e^{200 \pi}+1+\sin t$. By direct computation, we can obtain

$$
\begin{aligned}
& \bar{a}_{1}=2, \quad \bar{a}_{2}=4, \quad \bar{c}=1, \quad \overline{\left|a_{2}-b_{2}\right|}=2, \quad \bar{d}=50, \quad \bar{\beta}_{1}=\frac{1}{2 e^{200 \pi+1}}, \\
& \bar{\beta}_{2}=\frac{49}{e^{50} 3 \pi}, \quad \bar{e}=50 e^{200 \pi}+1, \quad \bar{b}_{1}=1, \\
& B_{1}=\ln \frac{4 \pi+2 \ln (2 / 3)}{2 \pi}+16 \pi+2 \ln \frac{2}{3} \doteq 50.07, \\
& B_{2}=\ln \frac{4 \pi-2 \ln (1 / 2)}{2 \pi}-16 \pi-2 \ln \frac{2}{3} \doteq-47.07, \\
& B_{6}=B_{27}=\ln \frac{100 \pi-2 \ln (3 / 4)}{2 \pi\left(50 e^{200 \pi}\right)}+200 \pi+2 \ln \frac{3}{4} \doteq-0.5754 ， \\
& B_{26}=\ln \frac{2 \pi+2 \ln (2 / 3)}{\pi / e^{200 \pi+1}} \doteq 629.55, \\
& B_{28}=B_{26}-200 \pi-2 \ln \frac{3}{4} \doteq 2.9362 \text {. }
\end{aligned}
$$

Then $B_{4} \doteq 50.07, B_{29} \doteq 2.9362$. It is easy to check that (5.1) satisfies all the conditions of Theorems 3.2 and 4.4 ; hence, (5.1) has a positive $2 \pi$ periodic solution which is global attractivity.

\section{Acknowledgments}

The authors would like to thank the referees for their helpful comments and valuable suggestions regarding this paper. This work is supported by the National Natural Science Foundation of China (no. 10771215), the Scientific Research Fund of Hunan Provincial Education Department (no. 10C0560), the Doctoral Foundation of Guizhou College of Finance and Economics (2010) and the Science and technology Program of Hunan Province (no. 2010FJ6021).

\section{References}

[1] F. Chen, "Almost periodic solution of the non-autonomous two-species competitive model with stage structure," Applied Mathematics and Computation, vol. 181, no. 1, pp. 685-693, 2006.

[2] F. Chen, "Periodicity in a ratio-dependent predator-prey system with stage structure for predator," Journal of Applied Mathematics, no. 2, pp. 153-169, 2005.

[3] F. Chen, "Positive periodic solutions of neutral Lotka-Volterra system with feedback control," Applied Mathematics and Computation, vol. 162, no. 3, pp. 1279-1302, 2005.

[4] S. Chen, T. Wang, and J. Zhang, "Positive periodic solution for non-autonomous competition LotkaVolterra patch system with time delay," Nonlinear Analysis: Real World Applications, vol. 5, no. 3, pp. 409-419, 2004.

[5] W. Ding and M. Han, "Dynamic of a non-autonomous predator-prey system with infinite delay and diffusion," Computers \& Mathematics with Applications, vol. 56, no. 5, pp. 1335-1350, 2008. 
[6] X. Ding, C. Lu, and M. Liu, "Periodic solutions for a semi-ratio-dependent predator-prey system with nonmonotonic functional response and time delay," Nonlinear Analysis: Real World Applications, vol. 9, no. 3, pp. 762-775, 2008.

[7] M. Fan, K. Wang, and D. Jiang, "Existence and global attractivity of positive periodic solutions of periodic $n$-species Lotka-Volterra competition systems with several deviating arguments," Mathematical Biosciences, vol. 160, no. 1, pp. 47-61, 1999.

[8] M. Fazly and M. Hesaaraki, "Periodic solutions for predator-prey systems with BeddingtonDeAngelis functional response on time scales," Nonlinear Analysis: Real World Applications, vol. 9, no. 3, pp. 1224-1235, 2008.

[9] K. Gopalsamy, "Global asymptotic stability in an almost-periodic Lotka-Volterra system," Australian Mathematical Society B, vol. 27, no. 3, pp. 346-360, 1986.

[10] V. Lakshmikantham, D. D. BaĬnov, and P. S. Simeonov, Theory of Impulsive Differential Equations, vol. 6 of Series in Modern Applied Mathematics, World Scientific Publishing, Singapore, 1989.

[11] Y. Li and Y. Kuang, "Periodic solutions of periodic delay Lotka-Volterra equations and systems," Journal of Mathematical Analysis and Applications, vol. 255, no. 1, pp. 260-280, 2001.

[12] X. H. Tang and X. Zou, "Global attractivity of non-autonomous Lotka-Volterra competition system without instantaneous negative feedback," Journal of Differential Equations, vol. 192, no. 2, pp. $502-$ 535, 2003.

[13] X. Tang, D. Cao, and X. Zou, "Global attractivity of positive periodic solution to periodic LotkaVolterra competition systems with pure delay," Journal of Differential Equations, vol. 228, no. 2, pp. 580-610, 2006.

[14] L.-L. Wang and W.-T. Li, "Periodic solutions and permanence for a delayed nonautonomous ratiodependent predator-prey model with Holling type functional response," Journal of Computational and Applied Mathematics, vol. 162, no. 2, pp. 341-357, 2004.

[15] W. Wang, G. Mulone, F. Salemi, and V. Salone, "Permanence and stability of a stage-structured predator-prey model," Journal of Mathematical Analysis and Applications, vol. 262, no. 2, pp. 499-528, 2001.

[16] X. Wu, J. Li, and Z. Wang, "Existence of positive periodic solutions for a generalized prey-predator model with harvesting term," Computers $\mathcal{E}$ Mathematics with Applications, vol. 55, no. 8, pp. 1895-1905, 2008.

[17] R. Xu, M. A. J. Chaplain, and F. A. Davidson, "Periodic solution of a Lotka-Volterra predator-prey model with dispersion and time delays," Applied Mathematics and Computation, vol. 148, no. 2, pp. 537-560, 2004.

[18] J. Yan and A. Zhao, "Oscillation and stability of linear impulsive delay differential equations," Journal of Mathematical Analysis and Applications, vol. 227, no. 1, pp. 187-194, 1998.

[19] S. J. Yang and B. Shi, "Periodic solution for a three-stage-structured predator-prey system with time delay," Journal of Mathematical Analysis and Applications, vol. 341, no. 1, pp. 287-294, 2008.

[20] Z. Yang and J. Cao, "Periodic solutions for general nonlinear state-dependent delay logistic equations," Nonlinear Analysis: Theory, Methods E Applications, vol. 66, no. 6, pp. 1378-1387, 2007.

[21] W. Zhang, P. Bi, and D. Zhu, "Periodicity in a ratio-dependent predator-prey system with stagestructured predator on time scales," Nonlinear Analysis: Real World Applications, vol. 9, no. 2, pp. 344353, 2008.

[22] W. Zhang, D. Zhu, and P. Bi, "Multiple positive periodic solutions of a delayed discrete predator-prey system with type IV functional responses," Applied Mathematics Letters, vol. 20, no. 10, pp. 1031-1038, 2007.

[23] Z. Zhang, Z. Hou, and L. Wang, "Multiplicity of positive periodic solutions to a generalized delayed predator-prey system with stocking," Nonlinear Analysis: Theory, Methods E Applications, vol. 68, no. 9, pp. 2608-2622, 2008.

[24] Z. Zhang, J. Wu, and Z. Wang, "Periodic solutions of nonautonomous stage-structured cooperative system," Computers E Mathematics with Applications, vol. 47, no. 4-5, pp. 699-706, 2004.

[25] Z. Zhang and X. Zheng, "A periodic stage-structure model," Applied Mathematics Letters, vol. 16, no. 7, pp. 1053-1061, 2003.

[26] L. Dong, L. Chen, and P. Shi, "Periodic solutions for a two-species nonautonomous competition system with diffusion and impulses," Chaos, Solitons and Fractals, vol. 32, no. 5, pp. 1916-1926, 2007.

[27] Y. Li, "Positive periodic solutions of nonlinear differential systems with impulses," Nonlinear Analysis: Theory, Methods E Applications, vol. 68, no. 8, pp. 2389-2405, 2008.

[28] Y. Li and L. Lu, "Global exponential stability and existence of periodic solution of Hopfield-type neural networks with impulses," Physics Letters A, vol. 333, no. 1-2, pp. 62-71, 2004. 
[29] Z. Liu and L. Chen, "Periodic solution of a two-species competitive system with toxicant and birth pulse," Chaos, Solitons and Fractals, vol. 32, no. 5, pp. 1703-1712, 2007.

[30] J. Shen and J. Li, "Existence and global attractivity of positive periodic solutions for impulsive predator-prey model with dispersion and time delays," Nonlinear Analysis: Real World Applications, vol. 10, no. 1, pp. 227-243, 2009.

[31] R. E. Gaines and J. L. Mawhin, Coincidence Degree, and Nonlinear Differential Equations, vol. 568 of Lecture Notes in Mathematics, Springer, Berlin, Germany, 1997.

[32] I. Barbălat, "Systèmes d'equations différentielles d'oscillations non linéaires," Revue Roumaine de Mathématique, vol. 4, pp. 267-270, 1959.

[33] T. Yoshizawa, Stability Theory by Liapunov's Second Method, Publications of the Mathematical Society of Japan, The Mathematical Society of Japan, Tokyo, Japan, 1966. 


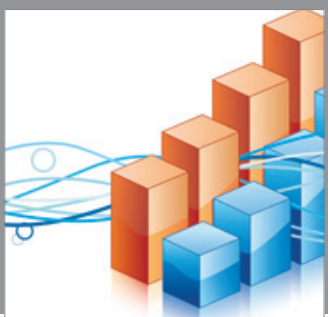

Advances in

Operations Research

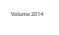

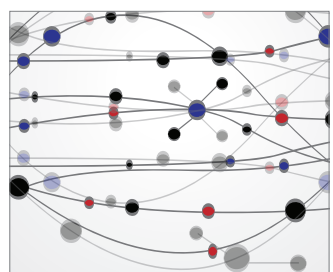

\section{The Scientific} World Journal
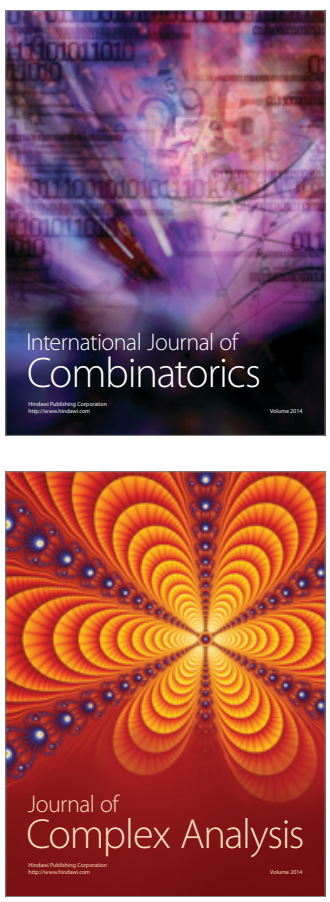

International Journal of

Mathematics and

Mathematical

Sciences
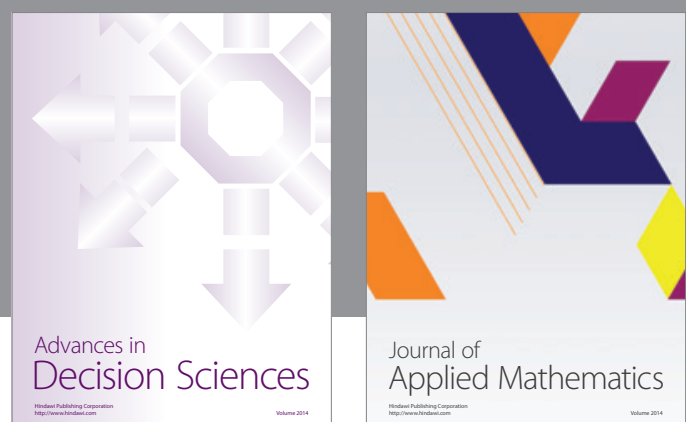

Journal of

Applied Mathematics
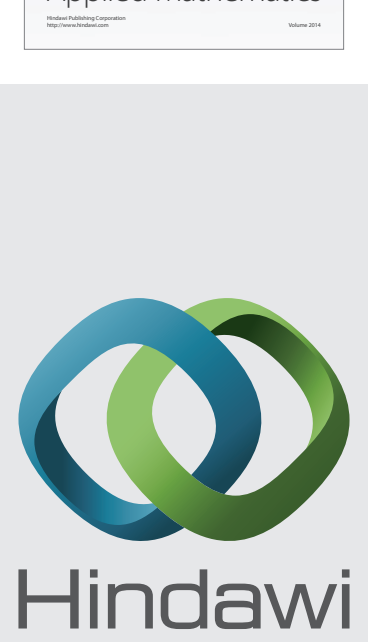

Submit your manuscripts at http://www.hindawi.com
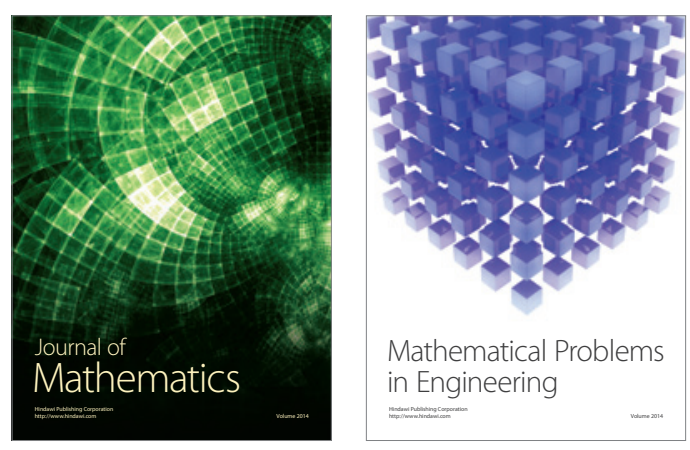

Mathematical Problems in Engineering
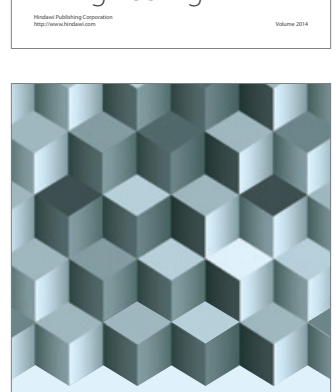

Journal of

Function Spaces
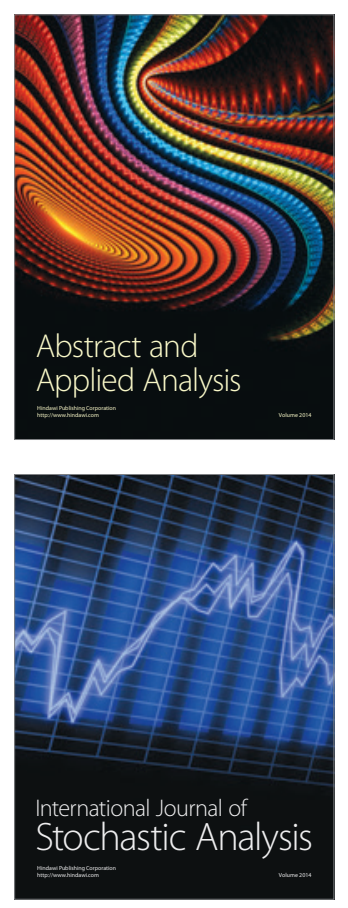

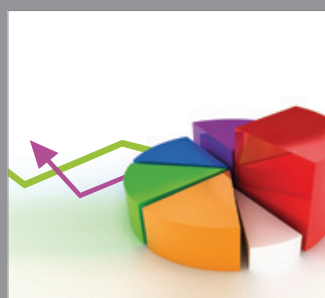

ournal of

Probability and Statistics

Promensencen
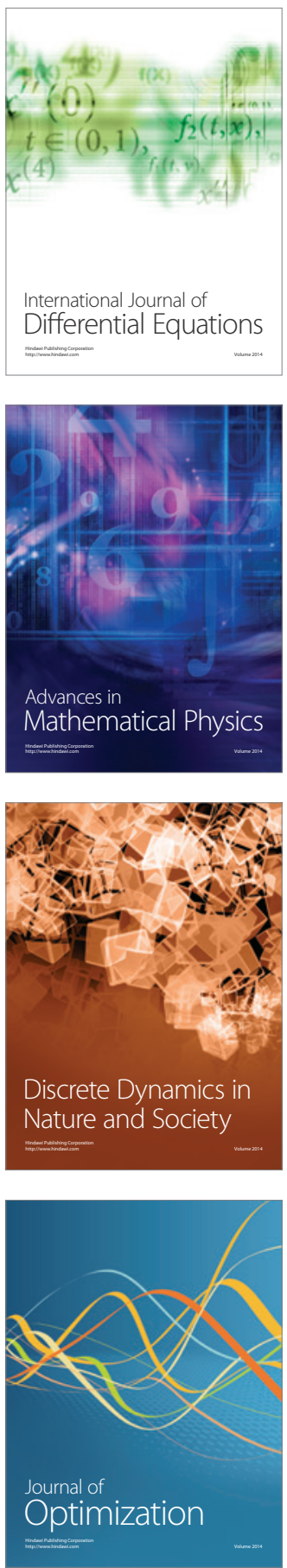